\title{
Recoilless gamma-ray lasers
}

\author{
George C. Baldwin and Johndale C. Solem* \\ Theoretical Division, Los Alamos National Laboratory, Los Alamos, New Mexico 87545
}

This review is addressed to the development of lasers that might generate coherent radiation at ultrashort wavelengths by stimulating recoilless nuclear transitions in solids. First, the authors review the basic physics of stimulated emission, superradiance and the kinetics of lasing, with particular attention to those aspects that characterize recoilless nuclear transitions in solid hosts. Then they classify the various approaches to pumping that have been proposed for resolving the "graser dilemma"- that the pump can destroy the conditions essential to gain-and discuss the general requirements for specification of an active nuclide and its solid host. The authors then classify and review those graser systems proposed since 1980 and prior to July 1996 in the published literature of the field, namely, (1) those that would pump directly, either with radiation or with intense bursts of neutrons; (2) those that would pump indirectly by first generating a nuclear isomer; (3) those that would eliminate the need for population inversion; and (4) several miscellaneous concepts. The significance of recent relevant experiments is described and discussed, and, finally, recommendations for future research are made. [S0034-6861(97)00504-7]

\section{CONTENTS}

I. Introduction

A. Purpose of this article

B. Why grasers?

C. Historical background

1. Conceptual overview

2. Previous reviews

a. Complete reviews

b. Focused reviews

D. Scope of this article

II. General Theory
A. Photon balance
B. Graser kinetics

1. General formulation

2. Coupled equations for field, populations, and transitions

3. Solution of the kinetic equations

4. Experimental confirmation

5. Relation to superradiance

C. Gamma-ray interactions in solids

1. Recoil from photon emission

2. Recoilless transition in solids

3. Linewidths of recoilless gamma radiation

a. Radiative width

b. Total linewidth

c. Typical magnitudes of line broadening

d. Control of linewidths

D. Loss processes

E. Crystals as resonators

1. The Borrmann effect

2. Wave modes in crystals

a. Two-wave Borrmann modes

b. Multiwave modes

III. Pumping
A. The graser dilemma
B. Grasers with unbound nuclei
C. Grasers with bound nuclei
1. Inversion by radioactive decay
2. Direct pumping

1086

1086

1086

1087

1087

1087

1087

1087

1087

1087

1087

1088

1088

1088

1089

1090

1090

1091

1091

1091

1091

1091

1092

1092

1092

1093

1093

1093

1093

1093

1094

1095

1095

1095

1095

1095

1096
*To whom correspondence should be addressed. a. Radiative pump 1096

b. Neutron pump 1096

3. Indirect pumping 1096

a. Two-step pumping 1096

b. Two-stage pumping 1097

4. Mixed-radiation pumps 1097

D. Pumping below inversion 1097

E. Isomer separation $\quad 1097$

IV. Materials for Grasers 1098

A. Essential nuclear requirements 1098

1. Level scheme 1098

2. Transition energy 1098

3. Lifetime range 1098

4. Competing reactions 1098

5. Availability and chemical compatibility 1098

B. Host candidates and properties 1098

1. Debye temperature 1099

2. Nonresonant absorption 1099

3. Crystal structure and stability 1099

4. Thermal properties 1099

5. Radiation damage 1099

V. Proposed Graser Systems 1099

A. Proposals related to pumping with neutrons 1099

1. Early proposals 1099

2. The proposal of Balko and Kay 1100

3. The Bowman proposal 1100

4. Analysis of Bowman's proposal 1100

a. Assumptions 1100

b. Method of analysis 1101

c. Parameter study 1101

d. Neutron moderation 1101

e. Conclusion 1102

5. Prospects for direct neutron pumping of
grasers

B. Proposals for two-step pumping 1102

1. Pumping with coherent radiation 1102

2. Pumping with incoherent radiation, e.g.,
bremsstrahlung

Mechanisms of interlevel-transfer pumping $\quad 1103$

a. Transfer by coherent radiation 1103

b. Transfer by incoherent radiation 1103

c. Preparing the storage isomer 1104

d. Candidate nuclides 1104

e. Difficulties 1105 
C. Proposals for stimulating long-lived transitions

1. Line narrowing

2. Inversion from radioactive decay

3. Homogeneous broadening by an NMR technique

D. Subinversion lasing: Proposals for suppressing resonant absorption

1. An optically pumped traveling-wave amplifier

2. Exploiting selection rules in a polarized population

3. Using RF to induce hyperfine transitions

4. Level-crossing concept

E. Miscellaneous concepts

1. Three-boson stimulated scattering

2. Nuclear Raman effect

3. Rapid heat removal under high pressure

4. Quasi-superradiance

VI. Experimental Work

A. Mössbauer effect in ${ }^{109} \mathrm{Ag}$

B. Time Filtering in crystals

C. ${ }^{125}$ Te experiments

1. Alpatov's experiments

2. Skorobogatov's experiments

3. Xu's experiment

D. Interlevel transfer

1. Search for multiphoton absorption in ${ }^{235} U$

2. The Texas experiments

E. Isomer separation and the ${ }^{197} \mathrm{Hg}$ experiments

1. Choice of isomer

2. Procedure

3. Results

4. Conclusions

VII. Recommendations

\section{A. Present status}

B. Approaches that merit further investigation

C. Approaches that lack promise

D. Approaches in which we see no merit

E. Research needed

1. Experimental

a. Isomer separation

b. Suppression of resonant absorption

c. Borrmann-mode Mössbauer experiments

d. Properties of candidate nuclides

e. Confirmation and extension of the Skorobogatov/Alpatov experiment

2. Theoretical
a. Subinversion lasing
b. Wave modes in crystals
c. Nuclear modeling
d. Thermal modeling
e. Nuclear superradiance
3. Concept elaboration

Acknowledgments

References

\section{INTRODUCTION}

\section{A. Purpose of this article}

An important goal of current laser research is to develop coherent sources for wavelengths below $1 \mathrm{~nm}-$ the gamma-ray or hard x-ray region. Subnanometerwavelength lasers could have many applications in basic science, technology, medicine, defense, perhaps even entertainment. ${ }^{1}$ In particular, they would have important applications in solid-state and nuclear physics and revolutionary impact in molecular biology. Controllable, intense beams of ionizing radiation with low diffraction spreading would be particularly attractive for strategic defense. Thus proposals to reach ultrashort wavelengths, most of them based upon the Mössbauer effect, have been appearing for over three decades, some promising, some duplicating concepts that were shown many years ago to be unworkable, some fallacious.

The graser remains a challenging but frustrating field of research, in which a considerable and rapidly growing literature now exists. Of the previous reviews, listed below, most relate work on one or a few of many proposed approaches; existing general reviews need to be updated. In this article we review work relating to the Mössbauer-effect approach that was published prior to mid 1996.

\section{B. Why grasers?}

Lasers for wavelengths down to about $10 \mathrm{~nm}$ use electronic transitions, featuring recombination of highly stripped ions in plasmas generated by bombarding line targets with intense pulses of radiation or particle beams. Extremely high pump intensity is necessary to reach the threshold for net gain, so it appears that further progress toward shorter wavelengths using this "brute force" approach will be very difficult-at least, so long as population inversion is an essential requirement.

Nuclear radiative transitions offer some relief from the high-pump-power approach, because excited states of nuclei commonly have lifetimes five or more orders of magnitude longer than electronic states of comparable energy, thus requiring far less power to excite a given density of excited states. Moreover, nuclear reactions, many of which are exoergic (in particular, reactions of neutrons from fission sources or accelerators), offer new approaches to pumping. One can envisage a solid laser medium, pumped by a nuclear reaction that generates an isomer, in which the Mössbauer effect greatly enhances the interaction of radiation with resonant nuclei, and the Borrmann effect reduces the parasitic absorption losses that normally reduce the gain. On the other hand, in gamma-ray emission, (1) protons, not electrons, are the radiating charges; (2) owing to the symmetrical structure of many nuclei, multipolarities of higher order than electric dipole are the rule; (3) electronic transitions in the atom (viz., internal conversion) often compete strongly with radiative transitions of excited nuclei; and (4) thermal motion and crystal imperfections broaden the gamma-ray line, weakening the resonance. Finally, and this is the most serious problem, the excitation process must not inhibit the Mössbauer and Borrmann effects, which are extremely sensitive to temperature and nuclear environment. These features make for

\footnotetext{
${ }^{1}$ More lasers are used in entertainment than for any other single purpose.
} 
a distinct kind of laser, differing fundamentally from present day optical and $\mathrm{x}$-ray lasers in physical characteristics and kinetic behavior.

\section{Historical background}

Speculation about nuclear-transition lasers began soon after the first demonstration of laser action in the optical band (Maiman, 1960).

\section{Conceptual overview}

Marcuse (1963) noted that recoil from the momentum of an emitted gamma ray reduces the overlap of absorption and emission lines and might permit gain without population inversion.

Others (Rivlin, 1961; Vali and Vali, 1963a, 1963b; Baldwin et al., 1963a, 1963b) noted that, since nuclei could be pumped by nuclear reactions, population inversion would not require the enormous pumping power that would be needed to invert atomic transitions of comparable energy. Gain might then be easily realized from nuclei bound in crystal lattices, because the Mössbauer effect would enable them to interact strongly with resonant radiation, while the Borrmann effect would greatly reduce the nonresonant (loss) photoelectric and Compton interactions. Still, they realized that not only are the conditions for recoilless resonance (Mössbauer effect) delicate, they can be destroyed by the pump, the so-called "graser dilemma."

Throughout the ensuing three decades, although this dilemma has defeated many otherwise ingenious concepts, new proposals continue to appear.

\section{Previous reviews}

For the reader who may want to research historical development in greater depth, we briefly list previous reviews here.

\section{a. Complete reviews}

These articles describe the entire area of stimulated gamma-ray emission up to their dates of publication, with particular emphasis on ideas that were considered new at the time. They are arranged chronologically.

Prospects for a gamma-ray laser (Baldwin and Khokhlov, 1975).

“The Gamma-Laser Problem" (Il'inskii, Yu. A., 1978, in Russian).

"Gamma-ray lasers?" (Gol'danskii, Kuz'min, and Namiot, 1981a), a book article.

"Approaches to the development of gamma-ray lasers" (Baldwin, Solem, and Gol'danskii, 1981).

"Grasers: proposals, problems and prospects" (Baldwin, 1982a).

"Gamma-ray lasers?" (Baldwin, 1982b).

"A critical review of gamma-ray laser proposals" (Baldwin, 1986).
"Gamma-Ray Lasers" (Elton, 1990). A chapter in a text on $\mathrm{x}$-ray lasers.

"Gamma-Ray Lasers" (Vysotskii and Kuzmin, 1989). A book, in Russian, published by Moscow State University.

\section{b. Focused reviews}

Several published reviews deal with one specific approach or a particular set of techniques.

"Multistep pumping schemes for short-wave lasers" (Baldwin, 1984). A book chapter.

"Coherent and incoherent upconversion schemes for pumping a gamma-ray laser" (Collins, 1986).

"Prospects for a gamma-ray laser based upon upconversion" (Collins, 1987).

"Nuclear Raman processes and the development of gamma-ray lasers" (Baldwin, 1989a).

"Status and Issues in the Development of GammaRay Lasers" (Collins et al., 1992).

"Progress in the Pumping of a Gamma-Ray Laser" (Collins et al., 1995).

"Recent Proposals for Gamma-Ray Lasers" (Baldwin and Solem, 1995b).

\section{Scope of this article}

The present review discusses approaches to graser development that would use recoilless nuclear transitions from nuclei bound in solid hosts; e.g., featuring the Mössbauer and, often, the Borrmann effect. Alternative approaches (viz., using nuclear transitions in gases, molecular beams or plasma or electron channeling in crystals) are not addressed.

Assuming that the reader is acquainted with the general features of nuclear gamma-ray emission, we begin by describing the kinetics of stimulated Mössbauer emission, noting the significant differences between conventional lasers and those that would use nuclear transitions. We then examine the factors that determine the gain, including, in particular, line breadth. The theory of crystal resonators for gamma radiation is briefly summarized. With this background, we can point out the essential requirements and practical limitations on the selection of suitable candidate nuclei and host crystals, and then proceed to classify the various concepts that are at present under consideration. These two sections will be largely qualitative.

In reviewing the currently popular concepts, we identify the most promising, in particular, the possibility of lasing without inversion. Lastly, we describe and comment upon the relatively small, but increasing, amount of experimental work that has been done to date in this largely speculative field.

\section{GENERAL THEORY}

\section{A. Photon balance}

It is common practice to estimate the performance of a graser system by employing a simple "photon- 
balance" relation between gain by stimulated emission and photon removal by absorption or scattering (Baldwin and Khokhlov, 1975),

$$
\frac{1}{\Phi} \frac{d \Phi}{d x}=\left(N_{2}-w N_{1}\right) \sigma-\mu,
$$

in which $\Phi$ is the photon current density ("flux"), $N_{2}$ and $N_{1}$ are the respective population densities of the lasant states, $w$ is the ratio of their statistical weights, $\sigma$ is the cross section for stimulated emission and/or resonance absorption, and $\mu$ is the linear photon removal coefficient.

When the populations are inverted above the threshold, i.e., when

$$
N_{2}>w N_{1}+\frac{\mu}{\sigma},
$$

this predicts an exponential growth of photon intensity in the lasing medium. Implicit in this relation is an assumption that stimulated emission is essentially instantaneous. For conventional lasers, which operate with greatly broadened transitions, usually by Doppler, this is satisfactory, but, in grasers that require recoilless transitions of near-natural linewidth (Solem and Baldwin, 1995), stimulated emission is not instantaneous, but evolves over a time of the order of the transition lifetime.

\section{B. Graser kinetics}

\section{General formulation}

A Mössbauer graser must necessarily be a single-pulse device, operating with a narrow emission line. Even if the pump were instantaneous, the inertia inherent in the response of any sharply resonant system would lead to behavior unlike that of conventional lasers, which have greatly broadened lines. For real pumping schemes, time is needed to populate the lasing states, and the populations change during the time required to establish the bandwidth. In particular, if the proposed pump is to employ slow neutrons, appreciable time is required to moderate and then capture them, so that an appreciably prolonged pump will result even from an essentially instantaneous fast-neutron source burst.

The interactions of electromagnetic radiation with atomic and/or nuclear systems are resonant processes. A general feature of all resonators is inertia of response to periodic perturbation. For example, the rise time and final amplitude of a forced oscillator circuit driven at its resonant frequency are inversely proportional to its damping factor; the quantum-mechanical analog to this classical process is resonance absorption. Classically, by reversing the phase of the perturbation one has resonance damping; its quantum analog is stimulated emission. No radiation is strictly monochromatic, and, by Fourier's theorem, a finite time is required to determine its frequency and bandwidth. Using Eq. (1) with a constant value for $\sigma$ ignores this inertia. Application of this principle to recoilless radiation occurs in the experiment of Lynch et al. (1960); its application to induced emission of recoilless radiation was first noticed by Chirikov (1963) and subsequently by Vorontsov and Vysotskii (1974) and by Solem and Baldwin (1995); its general application to lasers was discussed by Hopf et al. (1975), who coined the term "laser lethargy."

An accurate description of graser kinetics requires a set of coupled equations to account for the change in field amplitude and for the corresponding changes in population of the respective states from the pump, spontaneous decay, and the stimulated transitions.

We present the semiclassical kinetic theory, based on standard treatments of amplified spontaneous emission and superradiance given by many authors (Icsevgi and Lamb, 1969; MacGillivray and Feld, 1976) in calculations made for optical lasers, but in a form that is applicable to the special case of a brief-burst-pumped graser operating at near-natural linewidth (Baldwin and Solem, 1995a).

We assume that a monochromatic plane electromagnetic wave of the resonant frequency is injected suddenly into an extended medium that is being pumped uniformly throughout. The slowly varying amplitude of this wave is driven by displacement currents (variations of the nuclear charge polarization) in accordance with Maxwell's equations. We neglect retardation effects and treat the radiation-induced transitions between the two graser states quantum mechanically in the dipole approximation, to develop a formula for the nuclear charge polarization that drives the radiation field. We compute the population inversion density that appears in the polarization formula by integrating rate equations for the population densities of the parent nuclide and of the several states of the lasing nuclide that are involved in the pumping reaction. When combined by eliminating the polarization, these give a single differential equation for the amplitude $E(x, t)$ of a plane electromagnetic wave at the resonant frequency introduced with unit amplitude into the pumped medium at $(x=0, t=0)$.

\section{Coupled equations for field, populations, and transitions}

A monochromatic electromagnetic wave of frequency $\nu$ and electric-field amplitude $E_{0}$ propagates in a nonmagnetic medium with gain/loss in accordance with the equation

$$
\nabla \times(\nabla \times \vec{E})+\mu_{0} \epsilon \ddot{\vec{E}}=-\mu_{0} \ddot{\vec{P}}-\sigma_{e} \mu_{0} \dot{\vec{E}},
$$

where $\mu_{0}$ is the magnetic susceptibility of the vacuum, $\epsilon$ is the dielectric permittivity of the medium, and $\sigma_{e}$ is the electrical conductivity of the medium. An appropriate solution for the kinetics problem is a plane-polarized plane wave

$$
\vec{E}=\hat{z} E(x, t) \exp \left[2 \pi i \nu\left(t-\frac{x}{c}\right)\right],
$$

propagating in the $\hat{x}$ direction with a slowly varying envelope, 


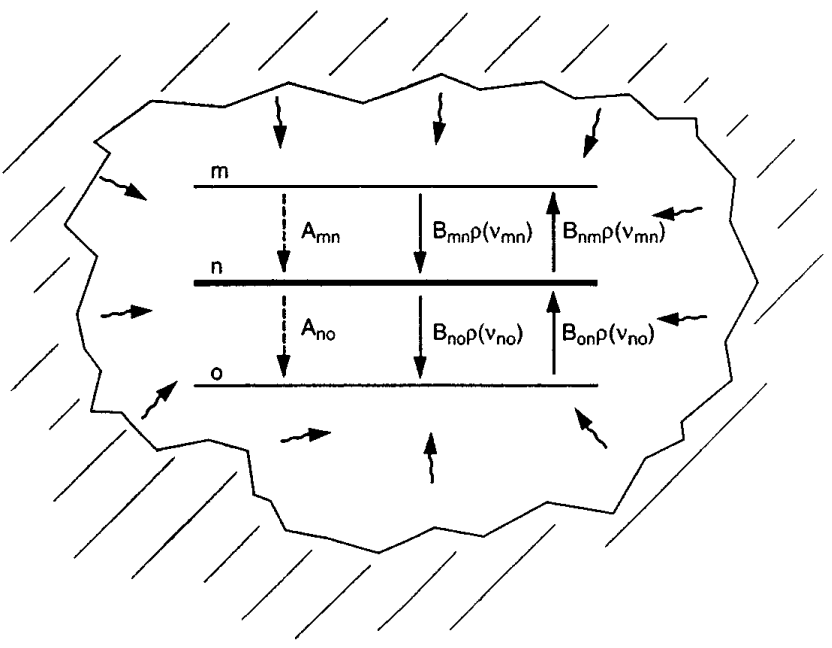

FIG. 1. Transition scheme for a three-level system in a radiation field. Level " $o$ " is the ground state; intermediate state " $n$ " is broader than the highest state " $m$ " because of shorter lifetime. Radiative transitions are labeled with their Einstein rate coefficients: $A$ for spontaneous, $B$ for transitions induced by the radiation field.

$$
\frac{\partial E}{\partial x} \ll \frac{\nu}{c} E, \quad \frac{\partial E}{\partial t} \ll \nu E, \quad \frac{1}{c} \frac{\partial}{\partial t} \ll \frac{\partial}{\partial x} .
$$

Substituting into Eq. (3), we obtain a relation for the wave amplitude in terms of the driving polarization,

$$
\frac{\partial E}{\partial x}+\frac{\mu}{2} E=-\frac{2 \pi \nu}{2 c \epsilon} P
$$

where we have introduced the absorption coefficient $\mu$ rather than expressing the attenuation in terms of conductivity.

Writing the time dependent Schrödinger equation in density-matrix form (Icsevgi and Lamb, 1969), we have

$$
\frac{i h}{2 \pi} \dot{\rho}=[H, \rho]
$$

where

$$
\rho=\left(\begin{array}{ll}
\rho_{n n} & \rho_{n o} \\
\rho_{o n} & \rho_{o o}
\end{array}\right),
$$

where the diagonal terms represent populations and the off-diagonal terms, transition moments (see Fig. 1). The macroscopic polarization is

$$
P=p\left(\rho_{\text {no }}+\rho_{\text {on }}\right),
$$

where $p$ is the nuclear polarization, and the inversion density is

$$
N^{*}=\left(\rho_{n n}-\rho_{o o}\right) .
$$

The density-matrix rate equations of the two-level system $(n \rightarrow 0)$ in an electromagnetic field are therefore

$$
\begin{aligned}
\dot{\rho}_{n n}(x, t)= & R_{2}-\frac{c}{\nu} \rho_{n n}-\frac{2 \pi i p}{h} E(x, t)\left(\rho_{n o}-\rho_{o n}\right), \\
\dot{\rho}_{o o}(x, t)= & R_{1}-\frac{c}{\nu} \rho_{n n}+\frac{2 \pi i p}{h} E(x, t)\left(\rho_{n o}-\rho_{o n}\right), \\
\dot{\rho}_{n o}(x, t)= & -\left(\frac{\gamma}{2 \pi}-2 \pi i \nu\right) \rho_{n o} \\
& -\frac{2 \pi i p}{h} E(x, t)\left(\rho_{n n}-\rho_{o o}\right), \\
\dot{\rho}_{o n}(x, t)= & -\left(\frac{\gamma}{2 \pi}-2 \pi i \nu\right) \rho_{n o} \\
& +\frac{2 \pi i p}{h} E(x, t)\left(\rho_{n n}-\rho_{o o}\right),
\end{aligned}
$$

where $R_{1}$ and $R_{2}$ are the pumping rates of the two levels and $\gamma$ is the decay rate of state $m$. The off-diagonal terms reduce to a differential equation that relates the polarization to the field amplitude,

$$
\frac{\partial P}{\partial t}+\frac{\gamma P}{4 \pi} E=-\frac{p^{2} E N^{*}}{h} .
$$

Combining Eqs. (6) and (12) to eliminate $P$ gives the kinetic equation

$$
\left(\frac{\partial}{\partial t}+\frac{\gamma}{4 \pi}\right)\left(\frac{\partial}{\partial x}+\frac{\mu}{2}\right) E=\frac{4 \pi^{2} \nu p^{2} E N^{*}}{2 \epsilon_{0} h_{c}} .
$$

If we substitute

$$
E=E_{0} \exp \left(-\frac{\gamma t}{4 \pi}-\frac{\mu x}{2}\right)
$$

into Eq. (13), we obtain the general kinetic equation

$$
\frac{\partial^{2} E_{0}}{\partial x \partial t}=\kappa E_{0}
$$

where

$$
\kappa=\frac{4 \pi^{2} \nu p^{2} N^{*}}{2 \epsilon_{0} h c}=\frac{\sigma N^{*} \gamma}{8 \pi} .
$$

The right-hand side of Eq. (16) contains all the relevant parameters of the resonant nuclear system.

3. Solution of the kinetic equations

A series solution to Eq. (15) is

$$
E_{0}=a_{0} \sum_{j=0}^{\infty} \frac{(\kappa x t)^{j}}{(j !)^{2}},
$$

a Bessel function of the first kind (Dwight, 1949). The amplitude of the field is then found to be

$$
E=a_{0} \exp \left(-\frac{\gamma t}{4 \pi}-\frac{\mu x}{2}\right) \begin{cases}I_{0}(2 \sqrt{\kappa x t}), & \text { if } N^{*}>0 ; \\ J_{0}(2 \sqrt{\kappa x t}), & \text { otherwise. }\end{cases}
$$

This differs significantly from the photon-balance result: stimulated-emission gain is not subtracted from (nor is resonant-absorption loss added to) the absorption coef- 
ficient $\mu$. This is a much lower order of dependence on distance than the exponential function expected from simple "photon balance" [see Eq. (1)] and its time dependence does not appear explicitly in the photonbalance relation. The first factor accounts only for ordinary decay and nonresonant absorption. The effect of resonant transitions is expressed in the Bessel function; in its argument, both time and distance occur symmetrically with a common coefficient.

\section{Experimental confirmation}

The damped (also called "time-filtered") pulse train found above is completely analogous to that observed in optical experiments from a freely decaying population inversion that has been suddenly created by an intense, brief laser pump (MacGillivray and Feld, 1976); in nuclear recoilless resonance, it was first confirmed experimentally in 1960 by Lynch and his colleagues, who derived a similar function [Eq. (18)] in an investigation of the time dependence of Mössbauer radiation (but from a steady source) transmitted by a resonant absorber foil (Lynch et al., 1960). Its relevance to graser kinetics was subsequently discussed by Chirikov, (1963) and by Vorontsov and Vysotskii (1974). Recent experiments demonstrating the effect are discussed in Secs. VI.A and VI.B.

\section{Relation to superradiance}

The radiative behavior of a graser system is completely described by these coupled Maxwell, Schrödinger, and pumped-population equations, so whether one chooses to label the train of delayed radiation pulses described by these functions as "amplified spontaneous emission," "superfluorescence," or "superradiance" is purely semantic. Their distinction refers to the way the radiating system is modeled and excited. Nevertheless, there is discussion in the graser literature that treats superradiance-the term coined by Dicke (1954) to describe coherent spontaneous emission from a manycomponent system-as a distinct phenomenon, a possible alternative to amplified spontaneous emission for generating coherent gamma radiation.

We shall not attempt to cover in this review the extensive literature on the general theory of superradiance; we refer the reader to the comprehensive review by Gross and Haroche (1982). Here we confine our remarks to the nuclear case.

As first pointed out by Dicke (1954), a closely packed population of two-level atoms (or nuclei) in a common radiation field can be treated as a single system that radiates coherently, rather than as an assemblage of independent radiators, whenever either (1) the dimensions of the system are small compared with the common wavelength or (2) a preferred direction exists, established by the history and/or structure of the system, into which the entire system can emit coherently. The atomic system is then characterized by two quantum numbers: $m$, the inversion number (difference between upperand lower-state populations), and $r$, a measure of the correlations among the radiating charges, defined with respect to the preferred direction. Dicke showed that the intensity radiated in that direction from such a system is

$$
I(\vec{k})=I_{0}(\vec{k})(r-m+1)(r+m),
$$

where $I_{0}(\vec{k})$ is the normal, incoherent intensity in the direction $\vec{k}$. In the special case of

$$
r=\frac{N}{2}, \quad m=\frac{N}{2},
$$

this can be, rather than directly proportional to the number $N$ of radiators, proportional to its square. He coined the term "superradiant" to describe such a state.

In the Dicke model, the interaction of radiation is with the entire system of atoms. The details of the interaction with individual atoms (viz., cross section, multipolarity, wavelength) are automatically included in the incoherent rate, $I_{0}(\vec{k})$.

In the decade after discovery of the Mössbauer effect, suggestions appeared (Kagan and Afanas'ev 1964; Podgoretskii and Roizen, 1960; Zaretskii and Lomonsov, 1965) that isomeric nuclei bound in crystals consisting of the same nuclide in their ground state should also be capable of coherent spontaneous emission and anomalous radiation rates. They reasoned that resonant absorption would spread the excitation throughout the crystal and that the crystal lattice would provide a reference direction. Terhune and Baldwin (1965) applied Dicke's model to a set of isomeric nuclei bound in a single-crystal lattice, for which the Bragg relation establishes a set of possible preferential orientations that fulfill the second condition. Introducing a term for the lattice vibrations (phonons) into the Hamiltonian for this system, they showed that the radiation rate in any direction satisfying the Bragg condition is given by Dicke's formula multiplied by the Debye-Waller-Mössbauer factor. From selection rules on $m$ and $r$, they concluded that a cascade of recoilless transitions from an initial fully inverted population of a nuclear isomer could develop a superradiant state. Those predictions may or may not have been confirmed (Sec. VI.C.2) by recent experiments (Skorobogatov and Dzevitskii, 1995). Trammel and Hannon (1975) concluded that superradiance is more probable than amplified spontaneous emission for an initially inverted system of bound nuclei. Baldwin and Feld (1986) applied formulas, derived from the coupled Maxwell-Schrödinger formalism in research on optical superradiance (MacGillivray and Feld, 1976), to estimate that $10^{13}$ excited nuclei are needed for superradiance to develop in hypothetical systems instantaneously inverted $\left({ }^{119} \mathrm{Sn},{ }^{133} \mathrm{Ba}\right)$ in hosts (diamond, $\mathrm{BN}$ ) of high Debye temperature.

However, none of these analyses addressed the practical problem of creating such an initial state of full inversion in a cool solid. Balko, Kay, and Neuberger (1995) calculated the pumping requirements for achieving superradiance in two cases: (1) pure ${ }^{60} \mathrm{Co}$, pumped by thermal-neutron capture in ${ }^{59} \mathrm{Co}$, which requires at 
least $10^{17}$ neutrons $\mathrm{cm}^{-2} \mathrm{~s}^{-1}$; and (2) ${ }^{119} \mathrm{Sn}$, pumped by electromagnetic radiation from the 245-day isomeric level via a postulated intermediate level to the upper Mössbauer level, which would require $10^{12} \mathrm{~W} \mathrm{~cm}^{-2}$.

Although population inversion occurs automatically in the case of a long-lived isomer that decays to a much shorter-lived state, the latter cannot accumulate an appreciable population, so that the simple Dicke treatment, for a two-level system in which the number of resonant atoms or nuclei is constant, may not apply directly. Nevertheless, recent experiments (Skorobogatov and Dzevitskii, 1995) indicate that, if a pure sample of one such isomer $\left({ }^{125 m} \mathrm{Te}\right)$ is incorporated in a solid host and cooled, there is some coherence in the spontaneous gamma radiation. See Sec. VI.C.2.

\section{Gamma-ray interactions in solids}

\section{Recoil from photon emission}

Momentum must be conserved when a nucleus emits or absorbs a photon. Energy must also be conserved, so the energy of an emitted gamma ray must be less than the nuclear transition energy, while that of an absorbed gamma ray must be more. If the nucleus is initially at rest, the recoil energy is

$$
R=\frac{E^{2}}{2 M c^{2}}=5.32 \times 10^{-4} \frac{E^{2}\left(\mathrm{keV}^{2}\right)}{A} \quad(\mathrm{eV}),
$$

where $M$ is the nuclear mass and $A$ is its mass number. The Doppler effect, in combination with the recoil from gamma-ray interaction, both broadens and shifts the gamma-ray line. Thus the photon energies from a gas or amorphous solid have a distribution

$$
p(\nu)=\frac{1}{\gamma_{D}} \sqrt{\frac{\ln 2}{\pi}} \exp \left[-\frac{4 \ln 2}{\gamma_{D}^{2}}\left(\nu-\nu_{0} \pm \frac{R}{h}\right)^{2}\right],
$$

where

$$
\gamma_{D}=2 \nu_{0} \sqrt{\frac{2 k T \ln 2}{M c^{2}}} \simeq \frac{\sqrt{R k T}}{h} .
$$

Here $\nu_{0}$ is the transition frequency and $T$ is the absolute temperature.

The recoil shift leads to an important difference between the nuclear and the optical range; in the latter, recoil is negligible in comparison with the thermal broadening. Inequality of emission and absorption energies suggests that a population inversion would not be needed for lasing in the nuclear range (Marcuse, 1963). However, at any practicable temperature, the Doppler breadth greatly exceeds the natural width of the gamma radiation, reducing the resonance cross section, and precludes lasing in any system of pumped nuclei except at impracticably low (microkelvin) temperatures. In this review, we consider only the recoilless case.

\section{Recoilless transition in solids}

The discrete vibrational ("phonon") spectrum of a solid with long-range order is determined by the mass of the atoms comprising the lattice, the strength of the bonds between these atoms, and the internal structure. Imparting a sufficiently large impulse (viz., from photon emission or absorption) to one of the constituent atoms will ordinarily change the occupation number of one or more of the vibrational states. However, if the energy of the impulse is small compared to the spacing of the vibrational spectrum, the occupation number may be left unchanged; the momentum then may be absorbed by a many-atom chunk of the solid acting as a unit, rather than by a single nucleus. Then the energy of the emitted gamma ray is very close to the nuclear transition energy, and, in general, the linewidth is comparable with the natural linewidth, and the emission is said to be "recoilless."

The probability that the gamma ray will be emitted without recoil is given by

$$
f=\exp \left[-\left\langle x^{2}\right\rangle\left(\frac{2 \pi \nu}{c}\right)^{2}\right],
$$

where $\left\langle x^{2}\right\rangle$ is the mean-square thermal fluctuation amplitude (Lamb, 1939; Mössbauer, 1958; Vischer, 1960). For a lattice that is very rigid compared to the recoil energy, $f \rightarrow 1$, and for a tenuous gas, $f \rightarrow 0$. For a Debye lattice,

$$
f=\exp \left\{-\frac{3 R}{2 k \Theta}\left[1+\left(\frac{2 \theta}{\Theta}\right)^{2} \int_{0}^{\Theta / \theta} \frac{x d x}{e^{x}-1}\right]\right\},
$$

where $\Theta$ is the Debye temperature.

Generally $f$ must be determined experimentally, although it is frequently convenient to describe its temperature dependence with an ad hoc Debye temperature (Frauenfelder, 1960), which may differ significantly from the Debye temperature that corresponds to the specificheat capacity. The Debye model has rather limited application to the Mössbauer effect, because most of the phonons exchanged in heat transfer have miniscule energies compared to typical recoil energies (Lipkin, 1960).

\section{Linewidths of recoilless gamma radiation}

\section{a. Radiative width}

Because of time-energy complementarity, the finite widths of excited nuclear states can be expressed in either energy or frequency (inverse time) units. We prefer to employ the latter convention, in which the "radiation width" of a state is the probability, per unit time, that the nucleus will undergo a radiative transition from that state, and is equivalent to the Einstein spontaneousemission coefficient $\left(\gamma_{r} \equiv A_{m n}\right)$. With alternative modes of deexcitation, the partial radiation width for recoilless emission can be written

$$
\gamma_{r}=\frac{2 \pi}{\tau_{m}} \frac{\beta f}{1+\alpha},
$$


TABLE I. Cases of line broadening.

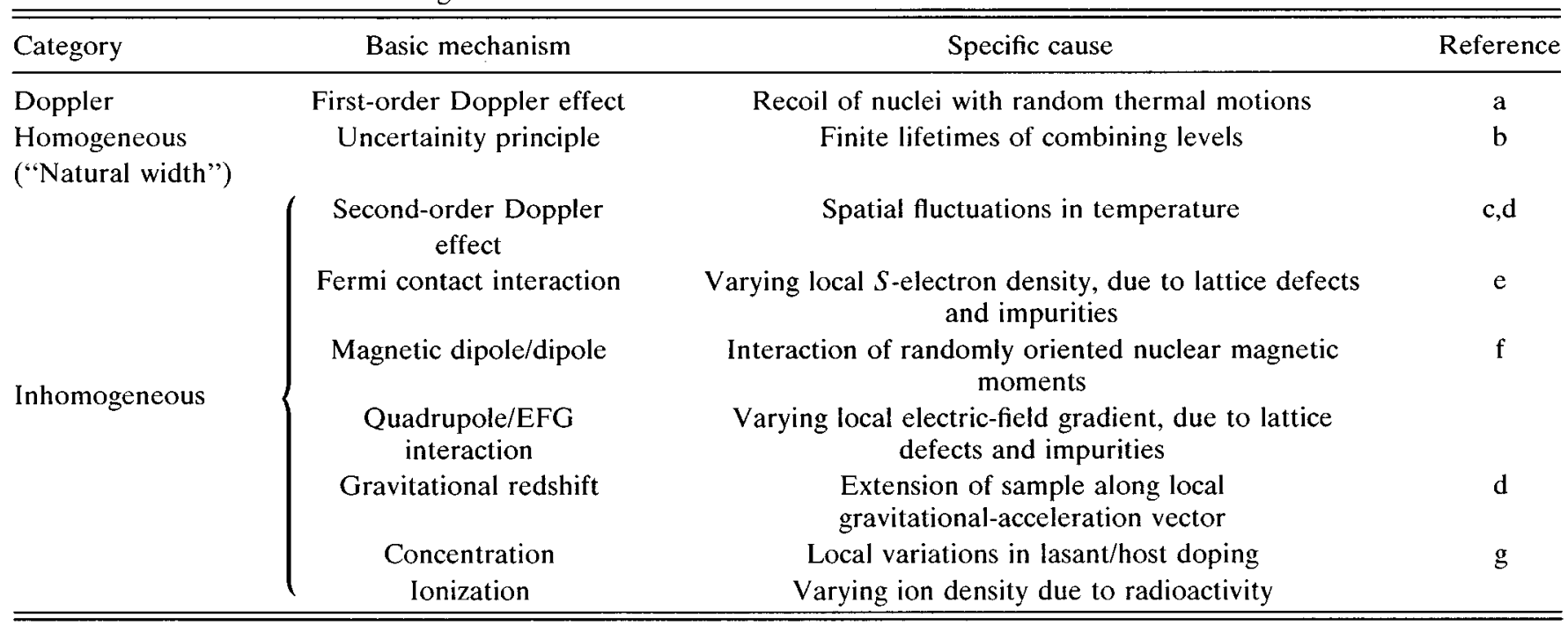

${ }^{\mathrm{a}}$ Frauenfelder, 1960

${ }^{b}$ Weisskopf and Wigner, 1930.

${ }^{c}$ Kagan, 1965.

dPound and Rebbka, 1960.

eShenoy and Wagner, 1978.

f Khokhlov and Il'inskii, 1973.

gVali and Vali, 1963a.

where $\beta$ is the branching ratio of the $m \rightarrow n$ transition to another level not shown in Fig. 1 , and $\alpha$ is the internal conversion coefficient. When the nucleus is bound in a solid host, one appends the recoilless fraction as a factor.

\section{b. Total linewidth}

Owing to nonradiative deexcitation processes, the total linewidth generally exceeds the radiative linewidth of the upper state, and therefore the resonance cross section is smaller than the wave cross section $\lambda^{2} / 2 \pi$. Any interaction in the host that randomly shifts the transition energy reduces the effective stimulation cross section. Lifetime (homogeneous) broadening associated with a decaying lower state, by reducing the ratio of radiative to total width, also often eliminates a nuclide from candidacy (Solem and Baldwin, 1995). We distinguish three types of broadening: (1) homogeneous, (2) Doppler, and (3) inhomogeneous. They are summarized in Table I.

\section{c. Typical magnitudes of line broadening}

Even the "best" Mössbauer transitions (to ground states) are perceptively broadened. Transitions from long-lived upper states, which would be easier to pump, are correspondingly more sensitive to such broadening (Khokhlov and Il'inskii, 1973).

Reviews of the origins and magnitudes of inhomogeneous broadening interactions in particular systems are given in Appendix $\mathrm{E}$ of Baldwin, Solem, and Gol'danskii (1981) and by Boolchand (1988). Boolchand's review stresses the importance of host-crystal structure and the extreme sensitivity of linewidth to common impurities; thus, for the narrowest lines, the crystal should preferably be cubic and substitutionally doped with the lasant atoms. Heroic measures are needed to approach the natural linewidth for Mössbauer transitions from states that decay to the ground state with lifetimes exceeding a few microseconds, for example, ${ }^{181} \mathrm{Ta},{ }^{73} \mathrm{Ge},{ }^{67} \mathrm{Zn}$. Experiments that have reported observable Mössbauer effects in even longerlived nuclei such as ${ }^{107} \mathrm{Ag}$ and ${ }^{109} \mathrm{Ag}$ will be discussed in Sec. VI.A.

\section{d. Control of linewidths}

Throughout the 1970s proposals were made for reducing or compensating selected inhomogeneous broadening effects by techniques that would employ radio frequency manipulation of magnetically aligned nuclei (Il'inskii and Khokhlov, 1973; Gol'danskii, Karyagin, and Namiot, 1974). In a previous review (Baldwin, Solem, and Gol'danskii, 1981), it was pointed out that during the minimum possible time needed for these techniques to restore the natural linewidth-given by the energy-time uncertainty relation-population inversion would be lost by decay. In view of the recent interest in subinversion lasing, it is timely to reexamine these proposals.

In a recent article, Balko and his collaborators (1995) have examined the effect of both homogeneous and inhomogeneous broadening and of nonresonant absorption on the development of superradiance by applying the theory of Haake and Reibold (1984) to the nuclear case, in order to estimate pumping requirements for two specific systems: (1) ${ }^{60} \mathrm{Co}$ pumped with thermal neu- 
trons; and (2) ${ }^{119} \mathrm{Sn}$ pumped from the 245 -day isomeric level by photons. Both cases required unavailable pump intensities. (Sec. III.A.2).

In as-yet-unpublished work, Balko and his collaborators consider the role of relaxation processes in reducing the broadening associated with inhomogeneous interactions, suggesting that fast relaxation may restore the natural linewidth. From a numerical study, they conclude that if the dephasing rate associated with the inhomogeneous perturbations is small, the superfluorescence can develop normally, but that there is a rapid deterioration of performance if the dephasing rate is comparable to the superfluorescence decay rate.

\section{Loss processes}

The photon-loss coefficient $\mu$ in the kinetic and photon-balance relations is the sum of the probabilities per unit distance of all processes that remove photons from the resonant bandwidth; i.e., the product of the "narrow-beam total" cross section for each element by its respective concentration. In the energy regime that permits recoilless nuclear resonance, these processes are principally photoelectric absorption and incoherent Compton scattering. Cross sections for these processes are tabulated in several references (Storm and Israel, 1967; Hubbell and Berger, 1965).

\section{E. Crystals as resonators}

From the earliest days of graser research, proposals have been made to use Bragg reflections in single crystals to achieve a resonant structure for gamma rays and thereby use a pumped medium more efficiently than is possible in a single-pass amplified spontaneous-emission system, just as Fabry-Pérot reflectors increase the efficiency of optical lasers. In particular, there were proposals for closed-path arrays of crystal reflectors alternating with pumped graser bodies (Deslattes, 1968) or for bending a pumped whisker crystal into a circle (Vali and Vali, 1963b). Such concepts present obvious practical difficulties. In more promising concepts, a single crystal of pumped material would act as a distributed resonator.

\section{The Borrmann effect}

In 1941, Borrmann (Borrmann, 1941) discovered that, when very-sharp-line characteristic $\mathrm{x}$ radiation is incident at a Bragg angle on a single crystal in the Laue geometry, the attenuation coefficient of the transmitted radiation is reduced by almost two orders of magnitude. The effect, independently rediscovered by Campbell (1951), had been predicted many years before by Ewald (1913). It results from the interference of Braggscattered waves within the crystal.

Early proposals recognized that this effect would be extremely beneficial in a graser (Baldwin, Neissel, Terhune, and Tonks, 1963a, 1963b), reducing the photon losses that compete with stimulated emission and thereby the threshold inversion density. If the crystal is

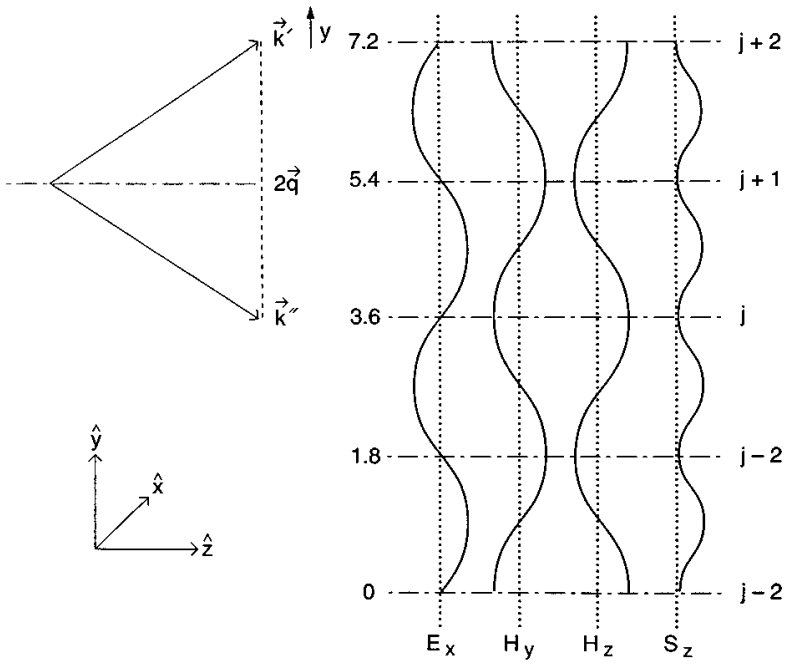

FIG. 2. Propagation vectors and electromagnetic-field amplitudes for the Borrmann mode in two-wave $m$ th-order Bragg diffraction from a set of lattice planes $y=\ldots(j-1) d, j d,(j$ $+1) \ldots$, as functions of position in the direct lattice (measured normal to the reflecting planes). Note that, in this mode, the electric-field amplitude and magnitude of the Poynting vector vanish, but that the electric-field gradient and the magnetic field have maxima at the lattice planes. In the anti-Borrmann mode (not shown), their phases are shifted by $90^{\circ}$.

cut in an acicular form with the direction of its greatest extent coinciding with the direction of propagation of a Borrmann mode, the crystal can function as a low-loss distributed resonator for that mode, with attendant reduction in the inversion-density requirements.

\section{Wave modes in crystals}

\section{a. Two-wave Borrmann modes}

In the simplest case, consider a beam of $\mathrm{x}$ or gamma radiation incident on a cubic crystal at the Bragg angle in Laue geometry with $s$ polarization. Assume that the lattice planes active in the Bragg reflection lie in the $x-z$ plane and have spacing $d$. Coherent ("Bragg") scattering by the atoms in these planes creates two sets of waves whose propagation vectors (Fig. 2) $\vec{k}^{\prime}$ and $\vec{k}^{\prime \prime}$,

$$
\vec{k}^{\prime \prime}-\vec{k}^{\prime}=2 q \hat{y},
$$

where

$$
q=\frac{m \pi}{d}=k \sin \theta,
$$

differ by a vector $2 q \hat{y}$ of the reciprocal lattice (James, 1950).

The vector potentials are, respectively,

$$
\begin{aligned}
& \vec{A}^{\prime}=\vec{x} A_{0} \exp \left[i\left(\omega t-\vec{k}^{\prime} \cdot \vec{r}\right)\right] e^{i \phi / 2}, \\
& \vec{A}^{\prime \prime}=\hat{x} A_{0} \exp \left[i\left(\omega t-\vec{k}^{\prime \prime} \cdot \vec{r}\right)\right] e^{-i \phi / 2} .
\end{aligned}
$$

Adding, we find the sum is a combination of two normal modes, 


$$
\vec{A}=\vec{A}^{\prime}+\vec{A}^{\prime \prime}=\hat{x} A_{0} \exp [i(\omega t-\kappa z)] \cos (q y-\phi / 2),
$$

where $\kappa=k \cos \theta$ and $\phi=0, \pi$. For one (the "Borrmann mode," $\phi=\pi$ ) shown in Fig. 2, the electric field vanishes at the lattice planes and, depending on the order of Bragg reflection, has one or more maxima in the spaces between them. The Poynting energy flow vector for this mode has the spatial dependence

$$
\begin{aligned}
& \kappa \sin ^{2}\left(\frac{m \pi y}{d}\right) \hat{z}-\left(\frac{m \pi}{d}\right) \sin \left(\frac{2 m \pi y}{d}\right) \hat{y}, \\
& m=0,1,2, \ldots,
\end{aligned}
$$

in which the first term describes propagation parallel to the lattice planes with maximum flow midway between them and none at the lattice planes, $y=m d$. The second term describes no net flow. Radiative interaction with the electronic structure within the crystal (photoelectric absorption and Compton scattering) is largely, but not entirely, electric dipole; with the nuclei, it is usually magnetic dipole, electric quadrupole, or even higher order. Therefore, although the Borrmann mode is attenuated only weakly by the atoms, to an extent depending on their finite extension and thermal motion measured by the Debye-Waller factor, it still interacts strongly with transitions of higher multipolarity in atoms (and nuclei!) at the lattice sites, because neither the magnetic vector nor the electric-field gradient vanishes at the reflecting planes-rather, they are maximum there. Thus, while the nonresonant electronic losses are reduced, recoilless nuclear resonance may, in fact, be enhanced if the active nuclei occupy lattice sites and the transitions are $M 1, E 2$, or higher order. Spontaneous emission from pumped nuclei then can excite, and stimulated emission can amplify, the Borrmann mode. The second normal mode, $\phi=0$, is rapidly attenuated by electronic processes.

\section{b. Multiwave modes}

Multiwave modes form if a sphere of radius $k$ may be passed through more than one pair of points of the reciprocal lattice (see Fig. 3.); i.e., if the Bragg condition holds simultaneously for more than one set of lattice planes. The $k$ vectors of the interfering waves define a cone.

The Rice University group (Hutton et al.) have pointed out that, in general, the larger $k$ and the greater the cone half-angle, the greater the number of different waves that can interfere, the sharper the resultant interference pattern, the stronger the interaction with the nuclei, and the weaker that with the electrons. Thereby, photon transmission losses (and thereby the threshold for net gain) are reduced still further, and, even more important, the slope of the gain-vs-inversion-density relation for excitation above threshold is greater than for the two-wave case. Moreover, higher beam multiplicity enhances the interaction with higher-order multipoles.

The $n$ superimposed waves within the crystal emerge as a set of $n$ separate beams directed along the surface
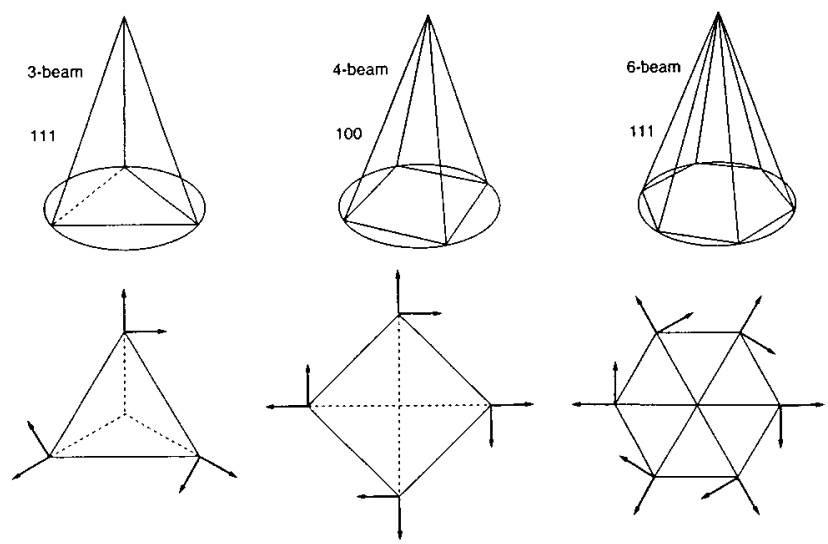

FIG. 3. Ewald-sphere constructions in the reciprocal lattice for multiwave interference in a crystal. The sphere of radius $k$ passes through $m$ points of the reciprocal lattice. The circles are traces of the wave emanating from the vertex that passes through points of the reciprocal lattice.

of a cone. Hannon and Trammell (1975) derived coupling factors to atomic electrons

$$
g_{A}=n \frac{F_{A}(\theta)}{\cos \theta}\left(\frac{4 \pi^{2}\left\langle x^{2}\right\rangle}{\lambda^{2}} \sin ^{2} \theta\right)^{p}, \quad p=1 \text { or } 2,
$$

and to the nuclei

$$
g_{n}=n \frac{F_{N}(\theta)}{\cos \theta},
$$

where $n$ is the number of interfering waves, $\theta$ is the cone half-angle, $\lambda$ is the wavelength, $\left\langle x^{2}\right\rangle$ is the mean-square displacement of atoms from lattice sites, and $F_{A}(\theta)$ $\left[F_{N}(\theta)\right]$ describes the multipole coupling of each beam to the atomic [nuclear] oscillator, $0 \leqslant F_{A}(\theta)\left[F_{N}(\theta)\right]$ $\leqslant 1$.

These formulas contain as factors (1) the multiplicity $m$, because of coherence, and (2) the secant of the cone half-angle, because the velocity at which the resultant wave advances is reduced (or the time of interaction is increased) by this factor. The factor $\left\langle x^{2}\right\rangle$, the meansquare displacement of the atom from the lattice site, occurs only in the electric dipole interaction. Figure 4, from Hutton's thesis, shows the product of the cosine of the cone half-angle with the coupling factor to magnetic dipoles of $m$-fold Borrmann modes. Table II compares coupling factors of ${ }^{57} \mathrm{Fe} / \mathrm{Fe}$ to two-beam and eight-beam off-Bragg Borrmann modes.

Multiple reflections within the crystal, like those of the Fabry-Pérot resonator of ordinary lasers, increase the interaction with the pumped nuclei; the crystal is an extreme case of a distributed-feedback resonator. Comparing multiwave modes in hypothetical Fe crystals doped with ${ }^{57 m} \mathrm{Fe}$ for $14.4-\mathrm{keV}$ Mössbauer radiation, Hutton (1988) found that multiplicities as high as 8 are possible (see Fig. 4), and the system should automatically select the largest allowable cone half-angle, for which the coupling factors are highest, for multibeam lasing. Hutton evaluated the enhanced gain coefficient for photon balance 


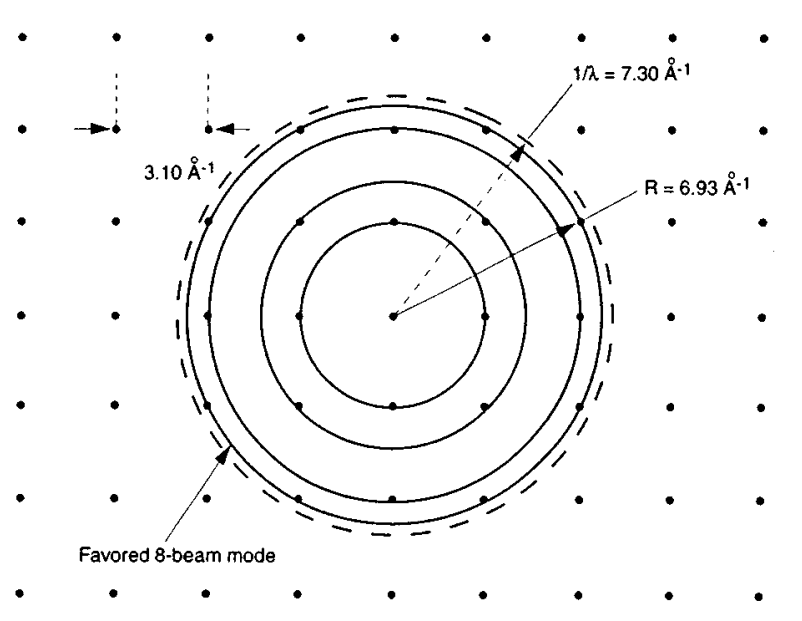

FIG. 4. Hutton's best case for ${ }^{57} \mathrm{Fe} / \mathrm{Fe}$, an eightfold multiwave mode.

$$
\kappa=\left(N_{2}-w N_{1}\right) g_{N} \sigma_{s}-g_{A} \mu,
$$

for ${ }^{57 m} \mathrm{Fe} / \mathrm{Fe}$ crystals. Figure 5 compares the gain coefficient of off-Bragg modes with the two-beam and eightbeam Borrmann modes as a function of inversion density.

Certain modes couple strongly to transitions of high multipolarity. In particular, Hutton describes a six-beam antisymmetric mode that couples strongly to octupole, but not to dipole or quadrupole transitions (see Fig. 6). Since a practical graser must operate at well above threshold excitation, multibeam Borrmann modes, which have (1) lower threshold inversion densities for net gain and (2) higher slopes of the gain-vs-inversiondensity curve for above-threshold excitation, can lower the practical requirement for the pump intensity by several orders of magnitude. Of course, it is still necessary that the pump not destroy the crystal integrity if the pumped crystal is to support such a highly ordered wave structure.

\section{PUMPING}

\section{A. The graser dilemma}

Creating a population inversion without destroying other conditions needed for gain is the outstanding difficulty in graser research.

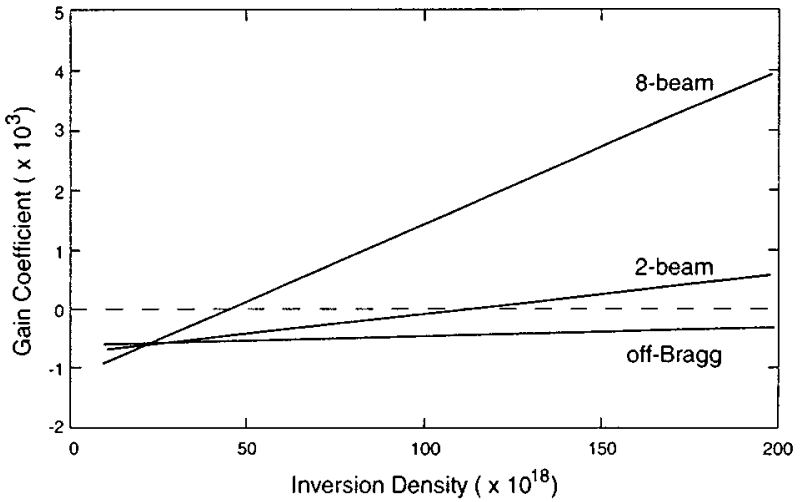

FIG. 5. Comparison of gain for off-Bragg, two-beam and eight-beam Borrmann modes as a function of inversion density.

\section{B. Grasers with unbound nuclei}

Attempts have been made to eliminate, not only the requirement of population inversion, but also the stringent requirements of the Mössbauer effect-in particular, that the graser medium be a cool solid-by reducing the recoil shift to less than the Doppler width, so as to eliminate the overlap of emission and nuclear resonance absorption lines. Although studies by Cohen (1988) and others of "non-Mössbauer" grasers have not been encouraging, Rivlin (1996) has suggested laser and accelerator techniques for cooling a beam of pumped ions. These proposals are outside the scope of this review, in which we treat only concepts that feature recoilless ("Mössbauer") transitions of nuclei bound in solids.

\section{Grasers with bound nuclei}

\section{Inversion by radioactive decay}

This class of concept would stimulate transitions from a long-lived ("isomeric") upper state $m$ to a shorterlived lower state $n$ (see Fig. 1). The isomer, prepared and concentrated radiochemically, would be introduced into a host solid where, whatever the initial mixture, faster decay of the lower state creates an inverted population. The solid would then be cooled to enable recoilless emission.

Discarded many years ago (Baldwin, Neissel, and Terhune, 1963a, 1963b; Vali and Vali, 1963, 1963b) as unworkable (Baldwin and Solem, 1994) because of homo-

TABLE II. Coupling factors of ${ }^{57 \mathrm{~m}} \mathrm{Fe} / \mathrm{Fe}$ to Borrmann modes (after Hutton et al., 1987).

\begin{tabular}{lcc}
\hline \hline Property & Two-beam mode & Eight-beam mode \\
\hline Cone angle, degrees & 1372 & \\
Radius of Ewald circle, $\mathrm{A}^{-1}$ & 6.7 & 23 \\
Nuclear coupling & 5.75 & \\
(relative to off-Bragg) & & 2.57 \\
$\begin{array}{l}\text { Electronic coupling } \\
\text { (relative to off-Bragg) }\end{array}$ & 1.38 & \\
\hline \hline
\end{tabular}




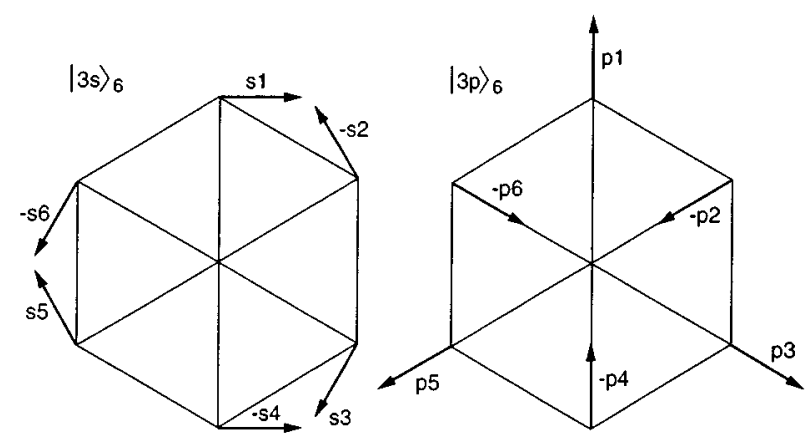

FIG. 6. Six-beam antisymmetric modes. These couple to $E 3$ or $M 3$, but not to dipole or quadrupole transitions.

geneous broadening, this idea is still occasionally revived (Skorobogatov, 1980, 1990, Greaves et al., 1987; Kamenov, 1988, 1991).

\section{Direct pumping}

In this category, the graser body is directly irradiated. The graser dilemma demands that the entire excitation energy be delivered into a very small volume in a time short compared with the lifetime of the upper state, which, in turn must be short to mitigate inhomogeneous broadening. The minimum number of nuclei that must be excited in a directly pumped graser [Eq. (20), p. 697 of Baldwin, Solem, and Gol'danskii (1981)] can be quite modest, of the order of $10^{13}$ ! Still, their concentration must be an appreciable fraction of the total atomic concentration of the graser body, and a concentrated, extremely efficient, highly specific pump is needed.

For example, a conceivable graser might comprise a cylindrical body $10^{-3} \mathrm{~cm}$ in diameter and $1 \mathrm{~cm}$ long, in which the pump supplies at least $20 \mathrm{keV}$ to each upperstate lasant nucleus of mean lifetime of not more than a microsecond. The corresponding stored energy density would exceed $3 \times 10^{4} \mathrm{~J} \mathrm{~cm}^{-3}$. The pump power density must exceed the corresponding decay power density, $20 \mathrm{GW} \mathrm{cm}^{-3}$; the host will inevitably have absorbed some of the pumping radiation, one cannot reach inversion by pumping directly from the ground state into the lasant state, the latter has been decaying during the pumping and lasing cannot begin instantaneously. The graser body would inevitably be destroyed-hopefully after the stimulated-emission pulse-if, indeed, such a pump could be made available.

\section{a. Radiative pump}

Consider, first, direct pumping with photons. Besides having relatively low cross section for nuclear excitation, photons are intrinsically inefficient. Except for characteristic $\mathrm{x}$ radiation, all sources of photons at the energies needed are broadband, calling for much higher power densities than those estimated above. The intense heating by nuclear recoil and the ionization that would be created within the graser solid by photoelectric and Compton processes are detrimental to maintenance of conditions for narrow-line recoilless emission.

\section{b. Neutron pump}

Slow neutrons have been proposed as pumps from the earliest days of graser research. Although they interact more strongly than photons with most nuclei and cause minimal lattice damage and heating in the solid host, they are also inefficient pumps, because the energy typically released in slow-neutron capture $(\sim 8 \mathrm{MeV}$ binding energy) exceeds that typical of Mössbauer transitions by at least two orders of magnitude.

Consider the case in which a pure parent nuclide is bombarded with a flux $\Phi$ of thermal neutrons. These neutrons are captured at a rate $\Phi \sigma_{c}\left(\mathrm{~s}^{-1}\right)$ to form the upper state, which decays with the rate constant $\lambda$ to form a lower state of the laser transition. From photon balance, Eq. (1), the minimum possible neutron flux is

$$
\Phi>\frac{\lambda \sigma_{a}}{\sigma_{s} \sigma_{c}},
$$

in which the subscripts on the various cross sections denote, respectively, $a$, nonresonant absorption; $c$, neutron capture; $s$, stimulated emission (asymptotic). Table III evaluates this for the case of ${ }^{56} \mathrm{Fe}(n, \gamma)$.

\section{Indirect pumping}

It is generally conceded that indirect pumping offers the best hope for pumping a graser to inversion without destroying the conditions for recoilless resonance. There are two categories of indirect pumping, designated as "two-step" and "two-stage," respectively.

\section{a. Two-step pumping}

In this category, the first step would be a slow process (viz., generating a long-lived "storage isomer" in a nuclear reactor or accelerator and concentrating it by radiochemical operations) that delivers most of the ex-

TABLE III. Pumping ${ }^{57 \mathrm{~m}} \mathrm{Fe}$ by thermal neutron capture in $56 \mathrm{Fe}$.

\begin{tabular}{lccc}
\hline \hline Property & Symbol & Value & Unit \\
\hline Decay constant, ${ }^{57 m} \mathrm{Fe}$ & $\lambda$ & $1.0 \times 10^{7}$ & $\mathrm{~s}^{-1}$ \\
Capture cross section, ${ }^{56} \mathrm{Fe}$ & $\sigma_{c}$ & 2.0 & barns \\
Removal cross section, $\mathrm{Fe}$ & $\sigma_{a}$ & 5500 & barns \\
Stimulation cross section, ${ }^{57 m} \mathrm{Fe}$ & $\sigma_{s}$ & 1.5 & $\mathrm{megabarns}^{-2}$ \\
Threshold neutron flux & $\phi_{\text {min }}$ & $2 \times 10^{28}$ & $\mathrm{~cm}^{-2} \mathrm{~s}^{-1}$ \\
Threshold neutron density & $\mathrm{n}$ & $8 \times 10^{22}$ & $\mathrm{~cm}^{-2}$ \\
\hline \hline
\end{tabular}


(a)

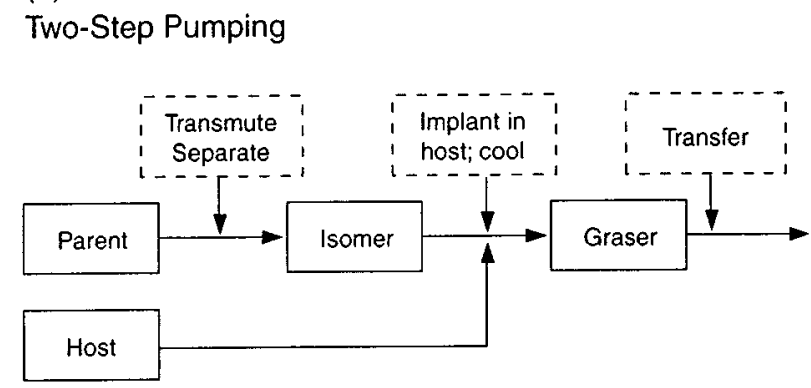

(b)

Two-Stage Pumping

\begin{tabular}{|c|c|c|c|c|}
\hline $\begin{array}{c}\text { Neutron } \\
\text { Source }\end{array}$ & Neutrons \\
\cline { 3 - 4 } & $\begin{array}{c}\text { Converter } \\
\text { Blanket }\end{array}$ & Resonant \\
Photons
\end{tabular} Graser

FIG. 7. Schematics of pumping: (a) Two-step pumping; (b) two-stage indirect pumping.

citation before the isomer is incorporated in host material to create the graser body. A second, fast pump, the "transfer step" would convert the storage isomer into the upper lasant state, with lasing to follow. Various proposals differ according to the identities of storage and lasant species, methods for generation, and separation of the storage isomer, graser geometry, and pump radiations (Fig. 7.)

\section{b. Two-stage pumping}

In two-stage pumping (Gol'danskii, Kagan, and Namiot, 1973), the first-stage ("primary") pump is thermalneutron capture in a "blanket" surrounding the graser body. The second stage is recoilless resonance absorption by the parent nuclide of a part of the neutron capture gamma radiation. This cannot create a population inversion between a pair of levels, so this recoilless transition must be to a third level higher in energy than the upper lasant level. There are few candidate nuclides, and for most of them insufficient data exist for quantitative description of the kinetic behavior and pump requirement. Analyses of this concept for ${ }^{57} \mathrm{Fe},{ }^{73} \mathrm{Ge}$, ${ }^{161} \mathrm{Dy}$, and ${ }^{181} \mathrm{Ta}$ (Baldwin and Solem, 1980) have been discouraging, particularly because of the unattainably high values of thermal neutron flux and the side effects (heating, lattice damage) of the capture gamma radiation.

\section{Mixed-radiation pumps}

Several proposals feature applying a mixture of radiations simultaneously, e.g., radio-frequency electromagnetic radiation along with the primary or transfer pump. Thus Karyagin $(1980,1995)$ suggests using radio frequency with applied magnetic fields to reduce the inhomogeneous broadening and polarize the nuclei, while pumping simultaneously with neutrons or photons.
Aside from complexity, the objections to direct pumps and to line narrowing alone suffice to make these proposals unpromising. Reiss (1986) suggested that intense radio-frequency fields, if used in conjunction with the transfer pump of a two-step operation, could supply additional angular momentum to reduce the "forbiddenness" of transitions of E3 and higher-order multipolarity. His proposal was later withdrawn after analysis by Friar and Reiss (1987) showed it to be infeasible.

Andreev et al. (1987) have analyzed the case in which a layer of active medium, deposited isomorphically on an undoped heat sink, recrystallizes after it has been melted, either by the pumping pulse (radiation unspecified) or by a simultaneous pulse of optical laser radiation. They assert that, because the time delay inherent in the development of superradiance (Sec. II.B.5) can exceed the thermal relaxation time, and because Borrmann mode formation in the annealed medium will greatly reduce the threshold for gain, superradiance can develop after the annealing. This, of course, assumes that the active material was not vaporized by the pump.

\section{Pumping below inversion}

If a practicable scheme for eliminating resonance absorption could be achieved, the threshold excitation level $\mu / \sigma_{s}$ for gain would be determined by the nonresonant photon removal coefficient, and the corresponding neutron flux would be a few orders of magnitude lower than that given in Table III.

\section{E. Isomer separation}

An indirectly pumped graser features a "storage isomer," of lifetime long enough for its activation, isolation from the parent and unwanted reaction products, purification, and incorporation in the host, possibly by epitaxial growth or ion implantation followed by annealing. Most proposals assume that the isolation step can be accomplished by (unspecified) radiochemical procedures. That would be true for the long-lived isomer ${ }^{93} \mathrm{Nb}$, which is formed by beta decay in fission product wastes in spent fuel from power reactor operation, but, in general, isomer separation is a serious problem that deserves further study than it has hitherto received. First, one must create the isomer, then separate it from other reaction products, including the ground state. The Szilard-Chalmers recoil-based technique (Baldwin, Neissel, and Tonks, 1966) can often concentrate the active isotope, but usually it still leaves a mixture of states.

Properties that distinguish the excited state of an isomeric nuclide from the ground state are energy (and, therefore, mass), nuclear volume (manifested by the chemical shift in Mössbauer lines), and usually (but not invariably) angular momentum. Typically, the relative mass difference is at most about $10^{-6}$, and so insufficient to provide a basis for separation from other forms of the same isotope.

On the other hand, hyperfine splitting of the resonance lines in the optical spectrum of the element, if sufficiently well resolved, may provide a basis for iso- 
merically selective excitation of nuclides whose isomeric and ground states have different angular momenta. After exciting to a higher state, with sharply tuned radiation, those atoms that have a chosen spectral component, it may be possible either to scavenge them with a chemical reagent (Baldwin et al., 1977) or to ionize them with a second radiation and collect the ions with an electric field (Dyer, 1986).

In a specific case that has been investigated experimentally (Sec. VI.E.1), even highly specific photoexcitation did not lead to a clean separation, because of exchange of excited with unexcited atoms before the former could be removed. Isomer separation-in particular, by atomic-beam methods for online separation of isomers created in accelerator targets-needs further research. There are other potential uses (Sharpe and Schmitt, 1959) for isomers besides the graser application.

\section{MATERIALS FOR GRASERS}

Many papers in the field contain tabulations of candidate nuclides; curiously, no two tabulations agree. Few papers address the other essential component, the host. Because of the great variety of proposed systems, none of which has yet been proved to be feasible, we do not offer another listing; rather, we discuss the general requirements.

\section{A. Essential nuclear requirements}

\section{Level scheme}

For two-step pumping, at least three energy levels are involved. The upper lasant state may lie either above or below, but must be very close in energy to the storage state; the lower lasant state may or may not be a ground state. Only two cases are known of pairs of levels in which the separation is of the order of a few electron volts: ${ }^{122} \mathrm{Th}$ and ${ }^{235} \mathrm{U}$. Finding, resolving, and measuring the separation of other such pairs is an extremely challenging problem, and theoretical modeling studies (Arthur and George, 1988) have not been encouraging. Alternative proposals for interlevel transfer with broadband energetic radiation, will be discussed in Sec. VI.D.2. Two-stage systems require a more complicated set of energy levels, e.g., more than one recoilless line.

\section{Transition energy}

For the recoilless fraction to exceed 0.1 , the freerecoil energy [Eq. (21)] must be less than about 1.5 times the effective Debye energy. This limits the gamma-ray energy to approximately

$$
E \leqslant 0.5 \sqrt{A \Theta}(\mathrm{keV}),
$$

where $A$ is the nuclear mass number. This is below 250 $\mathrm{keV}$ for any possible host/nuclide combination.

\section{Lifetime range}

Mössbauer transitions (i.e., to a stable lower state) with lifetimes ranging from nanoseconds to several microseconds have been identified in several score of nuclides. Since a graser can use transitions between excited states (with certain qualifications), there are many more potential candidates in that lifetime range.

Recoilless transitions can occur between states of longer lifetimes; the Mössbauer effect has been observed in ${ }^{107 m} \mathrm{Ag} / \mathrm{Ag}$ and ${ }^{109 m} \mathrm{Ag} / \mathrm{Ag}$, with lifetimes of the order of one minute, despite inhomogeneous line broadening, and in 58-day ${ }^{125} \mathrm{Te} / \mathrm{Be}$, despite its homogeneous broadening (Sec. VI). However, kinetic delays (Sec. II.B) should lead to very sluggish lasing.

Storage isomers must have lifetimes sufficient for generation, separation, concentration, and crystal growth or incorporation in the host material.

\section{Competing reactions}

Alternative reactions compete with the pumping reaction (viz., $(d, n),(d, p),(d, 2 n)$ in the production of ${ }^{197} \mathrm{Hg}$ by deuteron bombardment for the experiment on isomer separation described in Sec. VI.E). Internal conversion and branched transitions compete with the lasant transition, reducing the stimulation cross section.

\section{Availability and chemical compatibility}

An otherwise promising nuclide may be useless in practice because of low natural abundance, chemical instability, or incompatibility with a suitable host.

\section{B. Host candidates and properties}

Graser proposals that identify particular nuclides or particular pumps as suitable for the proposed system, but do not also specify the host and/or the system's geometry, are incomplete; the recoilless fraction is a function of properties of both the host and the lasant. A pure lasant material that can combine high recoilless fraction with low nonresonant attenuation is unlikely. Rather, the active lasant will be a minor constituent, in concentration only sufficient to establish useful gain; the graser body will be mainly host.

The criteria for a suitable host are at least as stringent as those for the lasant nuclide, and few candidates can meet them-especially if the solid is to provide a crystal resonator as well as to support recoilless emission. Most of the published work on the graser problem has focused on nuclear aspects (viz., identifying nuclides, level schemes, pumping reactions, and transition parameters), optimistically assuming that, once a suitable transition and pump have been identified, a host that will support recoilless emission would be available. Besides the effective Debye temperature and attenuation coefficient for the radiation to be stimulated, other important physical properties constrain the number of possible host/lasant combinations. 


\section{Debye temperature}

Tabulated Debye temperatures (Boolchand, 1988, p. 783 ) obtained from specific-heat data may differ appreciably from values that give the correct recoilless fraction when used in the standard formula, Eq. (25). Moreover, the effective Debye temperature for a host doped with a lasant of unequal nuclear mass is

$$
\Theta_{\mathrm{eff}}=\Theta_{D} \sqrt{M_{h} / M_{l}}
$$

where $M_{l}$ is the nuclear mass of the lasant and $M_{h}$ is the nuclear mass of the host.

\section{Nonresonant absorption}

Low- $Z$ materials [viz., lithium (Solem, 1979), beryllium (Gol'danskii and Kagan, 1973; Bowman, 1987), boron nitride, diamond (Baldwin and Feld, 1986), and silicon] are preferable for the host; the photon removal coefficient of the lasant-doped host determines the threshold inversion density.

\section{Crystal structure and stability}

Introduction of enough atoms of radioactive lasant into the host lattice to achieve gain must not render it unstable, change its structure, or unduly broaden the resonance. For multibeam interference (Sec. II.E), the graser body must be a single crystal, preferably cubic, with very few dislocations, and the lattice constant must exceed twice the gamma-ray wavelength.

\section{Thermal properties}

In a host material of high Debye temperature, the melting point will also be high, but it may not necessarily also have high thermal conductivity or diffusivity to enable rapid thermal relaxation during the brief period after the end of pumping while lasing is incubating. Examples of this consideration occur in analyses of a proposed superlattice geometry (Baldwin, 1988) and of a ${ }^{178} \mathrm{Hf} /$ diamond system pumped by bremsstrahlung (Davenloo et al., 1990).

\section{Radiation damage}

Besides heating, the pump can destroy the lattice perfection needed for a Borrmann mode. High Debye temperature usually ensures relatively high resistance to radiation damage. However, the only investigation of the collateral damage of pumping specifically directed to the graser field that we can find is a study of neutroninduced damage (Schwenn, 1978).

\section{PROPOSED GRASER SYSTEMS}

This section describes concepts for recoillesstransition grasers, with emphasis on those that have appeared since previous reviews, grouping them into a few general categories. In what follows, "lasant" designates the active nuclide, "upper" and "lower" refer to the two states of the transition to be stimulated; the "lower" may, or may not be, the ground state of the lasant.

\section{A. Proposals related to pumping with neutrons}

\section{Early proposals}

Proposals to use slow neutrons for direct pumping were the first to appear, and they continue to appear, because of their strong and usually exoergic nuclear interactions, ease of generation, and relatively low damage to crystal lattices. Fast neutrons, on the other hand, cannot be used as pumps because of unacceptable heating and damage to the solid host crystal of the graser.

As early as 1961 proposals appeared to create a longlived nuclear isomer [viz., ${ }^{104} \mathrm{Rh}$ (Vali and Vali, 1963a), ${ }^{115} \mathrm{In}$, or ${ }^{69} \mathrm{Zn}$ (Baldwin, Neissel, Terhune, and Tonks, 1963a)] by slow-neutron bombardment in a reactor, separate it, introduce it into a host solid, then cool it to raise the Debye-Waller factor to initiate stimulated emission (Rivlin, 1961). These ideas were abandoned because of unacceptable line broadening in isomers with lifetimes long enough for accumulating, separating, assembling, cooling, and stimulating before appreciable decay.

Gol'danskii and Kagan (1973) proposed to form the upper level of a short-lived recoilless transition, namely, the $6.3-\mathrm{KeV}$ state of ${ }^{181} \mathrm{Ta}$, by capture of neutrons from a nuclear explosive device by ${ }^{180} \mathrm{Ta}$ in a Be host. They calculated that unwanted radiation (capture gamma rays, photoelectrons) could escape from a long filament without overheating the host. A similar proposal by Solem (1979) suggested lithium as the host. Wood and Chapline (1974) advanced similar ideas.

Those proposals were based on simple photon balance. However, we have shown in Sec. II that, in a laser that requires recoilless emission, resonance inertia and decay of inversion reduce the ultimate value of the gain. Trammell and Hannon (1975) pointed out that including the kinetics would raise the neutron pump requirement by a factor of at least 20 .

Baldwin and Suydam (1977) reviewed the existing work on narrow-line graser theory and pointed out an additional problem, namely, that because all neutron source reactions generate fast neutrons, proposals to pump by capture of neutrons must also address the kinetics of moderating the neutrons. In a graser pumped by low-energy neutron capture, not only is time required for the stimulated emission to develop; an initially sharp burst of fast neutrons results in a delayed, sustained slow-neutron pump pulse of lower peak intensity.

Bowman (1976) suggested that ${ }^{83} \mathrm{Kr}$ in Be be pumped by both thermal- and resonance-neutron (40-eV) capture. A numerical study (Baldwin and McNeil, 1977) found that (1) neutron moderation time greatly exceeds the 141-ns mean lifetime of the ${ }^{83} \mathrm{Kr}$ transition and accounts for most of the delay before amplification begins; (2) broadening the Mössbauer transition reduces the kinetic delay in lasing, at the cost of reducing the stimulated-emission gain; and (3) most important, fastneutron source densities exceeding $10^{22} \mathrm{~cm}^{-3}$ would be 
required for appreciable amplification of the $9.3-\mathrm{KeV}$ spontaneous emission from this nuclide. Finally, Baldwin and Solem (1979a, 1979b) pointed out that it is thermodynamically impossible to generate such enormous thermal-neutron densities (Sec. V.A.5).

Nevertheless, two detailed proposals subsequently appeared in which intense bursts of neutrons would directly generate the lasant nuclide in a cobalt parent.

\section{The proposal of Balko and Kay}

Balko and Kay (1992) proposed to create ${ }^{60} \mathrm{Co}$ in ${ }^{59} \mathrm{Co}$ single crystals by the capture reaction ${ }^{59} \mathrm{Co}(n, \gamma){ }^{60} \mathrm{Co}$. They calculated that for superradiance to evolve in Borrmann modes in the Co host lattice, a thermal neutron flux of $2.1 \times 10^{23} \mathrm{~cm}^{-2} \mathrm{~s}^{-1}$ is required. This cannot be obtained from any existing neutron source. More recently, Balko, Kay, Silk, and Sparrow (1995) reexamined this case and found that, with realistic broadening values and possible values of thermal neutron flux, superradiance is unattainable in this system.

\section{The Bowman proposal}

Bowman (1987) has described what is probably the most detailed graser system yet proposed, with clever features addressed to practical problems of geometry, a system that it is possible to analyze in detail. Unlike earlier concepts that featured thermal neutron capture in the same element, it would be pumped by resonance neutrons. Bowman asserts that this reaction in ${ }^{57} \mathrm{Co}$ has a strong resonance at about $400-\mathrm{eV}$ neutron energy with a $7-\mathrm{eV}$ width and peak cross section of 300 barns. The parent nuclide ${ }^{57} \mathrm{Co}$ is radioactive, undergoing electroncapture decay to ${ }^{57} \mathrm{Fe}$, with a 270 -day half-life (Lederer and Shirley, 1978). For graser application, it must be carrier free.

Figure 8 labels the relevant energy levels of ${ }^{57} \mathrm{Co}$, ${ }^{58} \mathrm{Co}$, and ${ }^{57} \mathrm{Fe}$, with their energies, angular momenta, and lifetimes. $S$-wave capture of the neutron, followed promptly by emission of a proton, favors population of state 3, the $136-\mathrm{KeV}, 5 / 2$ - state of ${ }^{57} \mathrm{Fe} ; 89 \%$ of this state decays to the $14.4-\mathrm{keV}, 3 / 2$ level, state 2 , with only $11 \%$ going directly to the ground state, state 1 (Lederer and Shirley, 1978). Thus the $(n, p)$ reaction temporarily inverts the populations of states 2 and 1 of the lasing transition. Energy released in the $(n, p)$ reaction, shared between the emitted proton $(1.6 \mathrm{MeV})$ and the recoiling ${ }^{57 m} \mathrm{Fe}(22 \mathrm{keV})$, enables the ${ }^{57 m} \mathrm{Fe}$ to escape from a thin layer of parent ${ }^{57} \mathrm{Co}$ and embed itself in an adjacent layer of the host material.

Bowman therefore proposes to alternate thin laminae of ${ }^{57} \mathrm{Co}$ with laminae of $\mathrm{Be}$, and coil this multiple-foil "sandwich" into a tight spiral, so as to form a long cylinder-a nuclear jelly roll. Gamma rays emitted into the direction parallel to the spiral axis might then be amplified, if the magnitude and duration of the inverted ${ }^{57} \mathrm{Fe}$ population and of the recoilless fraction were sufficient.

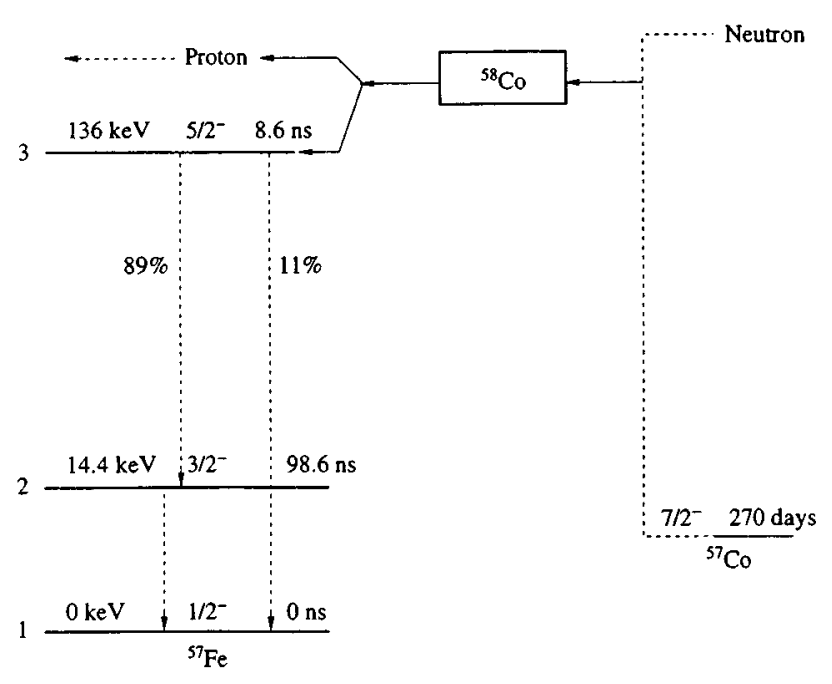

FIG. 8. Energy levels involved in Bowman's model of the pumping reaction (also applicable with little change to the Balko-Kay model). A neutron is absorbed by ${ }^{57} \mathrm{Co}$ producing highly excited ${ }^{58} \mathrm{Co}$, which by emitting a proton forms the $I=5 / 2+$ state of ${ }^{57} \mathrm{Fe}$. Most of those $136-\mathrm{keV}$ excited states decay to the $14-\mathrm{keV}$ excited state, which decays recoillessly to the ground state.

\section{Analysis of Bowman's proposal}

Bowman's otherwise complete paper implied that a spallation source might suffice, but gave no values for the minimum resonance-neutron fluence and pulse duration required for net gain, nor did it describe the kinetic behavior of this otherwise thoroughly designed system. Such an analysis, leading to a quantitative statement of the pumping requirement, is a necessary component of any feasible graser proposal.

To determine the pumping requirement, Baldwin and Solem made a numerical study, only to find that it, too, required an impossibly high neutron flux to create the necessary inversion. A full account has been published elsewhere (Baldwin and Solem, 1995b) but, because of its limited availability, we give a condensed version in what follows.

\section{a. Assumptions}

Baldwin and Solem assumed that (1) the extended medium is pumped uniformly throughout by a neutron burst; (2) destruction of the lasing states by the pump is negligible; (3) the neutrons are created in a square pulse of fluence $F$ and duration $T$, and with energies within the resonant bandwidth of the reaction that leads to inversion; (4) the pumping reaction directly populates only level 3 ; (5) all recoiling nuclei come to rest in the host layer immediately after they are created; (6) radioactive decay of the (initially pure) parent nuclide is neglected; (7) the radiation avalanche is initiated by spontaneous emission; and (8) the Mössbauer line is of natural width. These assumptions all overestimate the gain for a given pump fluence. 


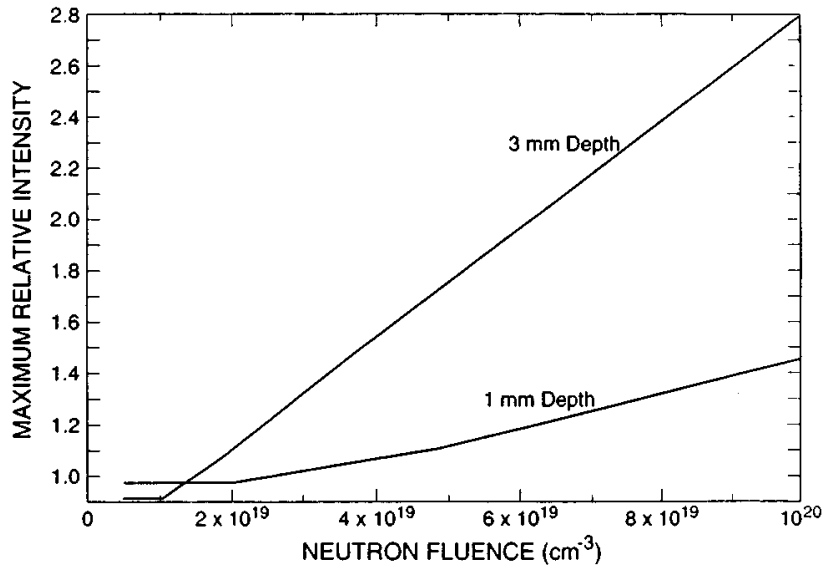

FIG. 9. Results of an analysis of the Bowman concept: gammaray intensities at 1- and 3-mm depths, for various neutron fluences delivered in 35-ns bursts. Enormous fluences are required for appreciable gain.

\section{b. Method of analysis}

The kinetic formulas derived in Sec. II.B.3, Eq. (18), give the temporal and spatial dependences of the amplitude of the traveling plane wave of resonant gamma radiation. The coefficient $\kappa$ in those formulas, containing the relevant properties of the resonant nuclear system, is time dependent through the inversion density $N^{*}$, which is determined mainly by the neutron pumping process, Eq. (16).

First, differential "rate" equations were written for the populations of each of the various species (e.g., Co parent, the $136-\mathrm{keV}, 8.6-\mathrm{ns}$ state, the $14.4-\mathrm{keV}, 98.6-\mathrm{ns}$ upper lasant state, and the stable ground state of ${ }^{57} \mathrm{Fe}$, taking account of destruction of the parent by the resonant neutron flux and of changes in the populations of the various iron states by interactions with neutrons and photons and by radioactive decay.

\section{c. Parameter study}

In a series of computations, Baldwin and Solem (1995b) assumed that a "square" burst of 400-eV neutrons of given fluence $F$ and duration $T$ bombards a "sandwich" target: a Be foil ("filling") $10^{-6} \mathrm{~cm}$ thick enclosed between two Co layers $2.5 \times 10^{-6} \mathrm{~cm}$ thick, as given in Bowman's original proposal, and calculated the time-dependent population densities of the respective states, the photon attenuation coefficient (which increases as Fe implants into the Be host), and the resultant intensities of the $14.4-\mathrm{keV}$ radiation at two different distances from a spontaneous source, for a succession of values of neutron fluence and burst duration.

They found that only with a pump flux in excess of $10^{27} \mathrm{~cm}^{-2} \mathrm{~s}^{-1}$ is the gain even barely adequate to overcome spontaneous emission. Making the pump burst longer than the optimum value of $T=35 \mathrm{~ns}$ is detrimental, because once ${ }^{57} \mathrm{Fe}$ ground states have begun to accumulate, inversion terminates rapidly while nonresonant absorption continues to increase.

Figure 9 summarizes calculations with varied fluence

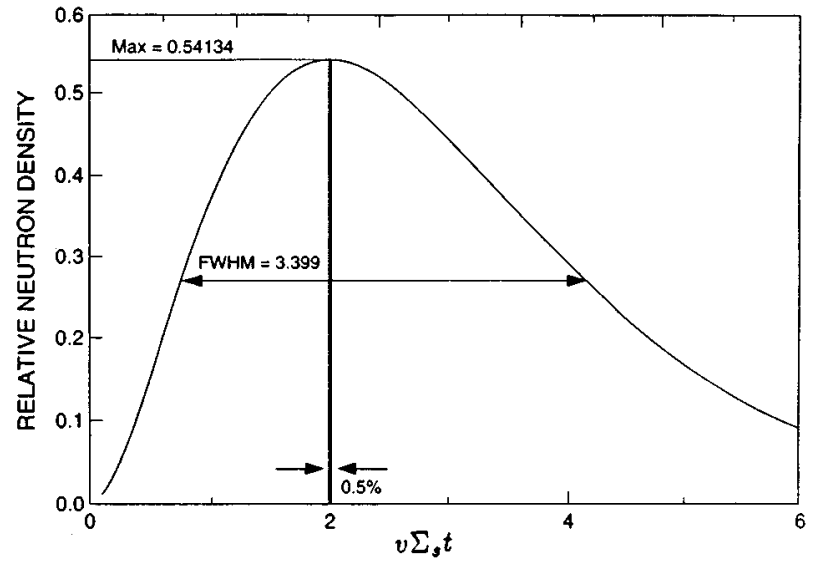

FIG. 10. Universal function for the density vs time-velocity spectrum of neutrons released at $t=0$ at infinite energy and in unit density, into an infinite hydrogenous moderator, neglecting capture (Williams, 1966). The independent variable $v \Sigma_{s} t$ $\mathrm{cm}^{-1}$ is the product of velocity, scattering probability, and time.

$F$ and a fixed pump duration of $T=35 \mathrm{~ns}$. A resonanceneutron fluence exceeding $5 \times 10^{18} \mathrm{~cm}^{-2}$ is needed for amplification even barely to exceed losses by decay and absorption at $3 \mathrm{~mm}$ from a spontaneous source. The actual pumping fluence needed for gain is far greater, because the energies of these neutrons must all lie within the 7-eV resonant bandwidth of the $(n, p)$ reaction, so their velocities must be in the range $v=2.77 \pm 0.013$ $\times 10^{7} \mathrm{~cm} \mathrm{~s}^{-1}$, and their density is $n=F / v T=10^{19} \mathrm{~cm}^{-3}$.

\section{d. Neutron moderation}

Because faster neutrons, if present, will have only deleterious effects, we must examine the spectrum of the moderated neutrons. In the ideal case, a burst of $n_{0} \mathrm{~cm}^{-3}$ neutrons at very high initial energy is suddenly injected at $t=0$ throughout a cold hydrogen moderator of infinite extent, afterwards moderating without capture to velocity $v$ by elastic scattering with probability ("macroscopic cross section") $\Sigma_{s} \mathrm{~cm}^{-1}$, the total neutron density $n$ and the moderator temperature remaining constant. Figure 10 shows their spectrum, given by the universal formula (Williams, 1966)

$$
n(\sigma, t) d v d t=n_{0}\left(v \Sigma_{s} t\right)^{2} \exp \left(-v \Sigma_{s} t\right) d v d t,
$$

in which velocity $v$ and time $t$ enter symmetrically as a product with the neutron-proton collision probability, $\Sigma_{s}$. When $400-\mathrm{eV}$ resonant neutrons are at their maximum density $\left(v \Sigma_{s} t=2\right)$, the number within the $7-\mathrm{eV}$ resonant bandwidth is a fraction

$$
\frac{\Delta n}{n}=\int_{1.995}^{2.005} x^{2} e^{-x} d x=0.005
$$

of all the neutrons at $35 \mathrm{~ns}$ after injection. Not only must the initial neutron density exceed by at least two orders of magnitude that of the neutrons that will do the pumping; the average time for fast neutrons to moderate to $400 \mathrm{eV}$ has an uncertainty spread of $90 \mathrm{~ns}$, far more than the optimum burst duration found above. 
Williams's formula neglected heating of the moderator by the neutrons. Their energies cannot be transferred to the moderator after equipartition has been reached. Simple heat balance (Baldwin and Solem, 1979a) shows that the maximum density of neutrons that can moderate from $2.5 \mathrm{MeV}$ to $300 \mathrm{eV}$ in an infinite hydrogenous moderator is only about $5 \times 10^{18}$; real, necessarily finite moderators would yield a far lower density and require a longer time.

\section{e. Conclusion}

We conclude that it is impossible for any known neutron source to deliver the resonant neutron flux required to pump the ${ }^{57} \mathrm{Co}$ system to a lasing state.

\section{Prospects for direct neutron pumping of grasers}

Bowman's concept, which employs a transition with the strongest known Mössbauer effect, an enriched parent with uniquely favorable nuclear parameters, a rigid low-absorption host, and clever design features, should require less neutron density than any other graser concept that features direct neutron pumping.

Moreover, even if enough resonant neutron flux for the Bowman concept or thermal neutron flux for the Balko-Kay concept could be delivered, it would be necessary to prevent those other interactions of the moderating neutrons (e.g., scattering on the active nuclei, transmutation of the host, lattice damage, heating) that destroy the Mössbauer/Borrmann conditions.

We conclude that, in general, direct neutron pumping cannot create a population inversion.

\section{B. Proposals for two-step pumping}

In most recent concepts based on the Mössbauereffect approach, a long-lived "storage" isomer, prepared by a conventional nuclear reaction, is separated from the reaction mixture, incorporated in a crystal host, and then exposed to a burst of radiation that would transfer its excitation from the storage state to another state, of short lifetime, from which stimulated emission to a still lower level can occur. In this way, most of the energy of excitation could be supplied before assembly of the graser body, and the second pump would create less heat and damage. Such "two-step" concepts have been proposed from the late 1960s (Eerckens, 1969).

Some general considerations relating to two-step systems were pointed out long ago (Baldwin, Solem, and Gol'danskii, 1981; Baldwin, 1982b). Since the storage and lasant levels must be close in energy, preferably within a few electron volts of each other, yet have greatly different lifetimes (and, therefore, different linewidths and angular momenta) the cross section for transfer will necessarily be small. Those concepts that are still actively considered fall into two subcategories, according to whether transfer radiation is to be coherent or incoherent.

\section{Pumping with coherent radiation}

Tunable, monochromatic, coherent laser radiation would be an ideal transfer pump, because (1) it could be focused precisely on the slender graser filament, (2) a multiphoton process could fulfil the angular momentum requirements, (3) no unwanted wavelengths would be present, hence (4) it would be uniquely efficient. For references to early proposals, see Solem (1986a).

In order to reduce lattice heating and damage, Baldwin (1988) proposed to distribute the isomer isomorphically at regularly spaced regions so as to comprise a superlattice within the crystal host, into which monochromatic and coherent transfer radiation would be introduced at a Bragg angle. Thereby, both the longwavelength pump and the stimulated gamma radiation could form Borrmann modes (Sec. II.E.2). He claimed that this would (1) maximize both nuclear interactions at the active nuclei; (2) enable the undoped regions of the host to act as heat sinks; (3) enable many graser filaments to lase simultaneously, and thereby (4) avoid the limitation on graser volume imposed by short wavelength and low Fresnel number. Unfortunately, no suitable nuclide that provides a closely spaced storage and lasant pair of levels has been identified, despite a careful search of available databases, nor has an isomer yet been deexcited by laser irradiation [Sec. IV.A].

\section{Pumping with incoherent radiation, e.g., bremsstrahlung}

$\mathrm{X}$ rays have often been proposed as pumps. Characteristic (line) x rays (Letokhov, 1973; Vysotksii, 1979) have been considered, but an exact energy match with a nuclear transition is extremely improbable. Olariu et al. (1982) described a special case of radiative pumping; as discussed in Sec. III.C.2.b, it would require so much pump power that the graser dilemma defeats it.

Undeterred by the graser dilemma, Collins et al. advocate a scheme in which intense $\mathrm{x}$ radiation would pump the active nuclei into their lasant state, either from their ground state (as in the ruby laser) or from a previously separated storage isomer (Collins, 1991), via a broad, much higher "gateway state." They have demonstrated (Collins and Carroll, 1995) that such a broad state does exist and that intense, broadband, incoherent radiation-bremsstrahlung from an accelerator-can indeed transfer population, in particular, from the naturally occurring isomer ${ }^{180 m} \mathrm{Ta}$ to its ground state, despite an angular momentum change of 8 units! (Sec. VI.D.2). They have also shown that they can excite nuclear levels that could be the upper states of grasers, by way of broad, much higher gateway states, and they have listed a number of candidate nuclides for this approach.

Collins and co-workers claim that the graser dilemma does not defeat this approach in a system that requires recoilless transitions and Borrmann modes, by a numerical estimate (Collins and Carroll, 1995) employing very optimistic assumptions (Sec. V.B.3.e). 


\section{Mechanisms of interlevel-transfer pumping}

\section{a. Transfer by coherent radiation}

An early concept (Eerckens, 1969) was to use a resonant electronic transition to drive the nuclear transition between the storage state and the upper state, a process analyzed by Morita and termed by him "NEET" (nuclear excitation by electronic transition) (Morita, 1973). This process was invoked (Izawa and Yamanaka, 1979) to account for the excitation of $75-\mathrm{eV}{ }^{235 m} \mathrm{U}$ from the ground state of ${ }^{235} \mathrm{U}$ in a laser-produced plasma, although that experiment could be alternatively explained by the capture of a free electron from the continuum into an atomic-shell vacancy with transfer of recombination energy to the nucleus (Gol'danskii, Kuz'min, and Namiot, 1981b).

Rather than shielding the nucleus, the atomic electrons might assist in the process by providing a large antenna, well matched to the laser light, whose energy could then be transferred via the near-field interaction to the small antenna of the nucleus (Solem, 1985, 1986a, 1986b; Biedenharn, Boyer, and Solem, 1985, 1986; Solem and Biedenharn, 1988). Nuclear interlevel transfer by coupling either to the nucleus or to a single electron is restricted to dipole transitions at the quantum energy of the driving laser, and the number of states addressable by the quantum energy band available from ordinary lasers is rather limited. If a long-lived state were energetically close to a short-lived state, the angular momenta of the two states must differ by several units. For example, ${ }^{235} \mathrm{U} \rightarrow{ }^{235 m} \mathrm{U}$ is an octupole transition involving a change of three units of angular momentum. Direct dipole transition between the two states would be highly forbidden, although intense lasers of the correct frequency might be able to induce transfer by multiphoton interaction. But Berger et al. (1988) calculated the probability of the ${ }^{235} \mathrm{U} \rightarrow{ }^{235 m} \mathrm{U}$ nuclear transition, first treating the atomic electrons as a gas and later (Berger et al., 1991) in a quantum-mechanical model in which electrons, moving in the combined Coulomb field of the nucleus and the sinusoidal laser field, could perturb the nucleus. They found negligible transition probabilities even at irradiance as high as $10^{21} \mathrm{~W} \mathrm{~cm}^{-2}$. See Sec. VI.D.1 for related experimental work.

An attractive alternative mechanism is suggested by the experiments of Rhodes et al., who, using ultra-highintensity $\left(10^{16}\right.$ to $\left.10^{17} \mathrm{~W} \mathrm{~cm}^{-2}\right) \mathrm{KrF}$ laser pulses, have observed the expulsion of inner-shell electrons, which they ascribe to collisions with a collectively oscillating outer shell (Boyer and Rhodes, 1985; Rhodes, 1985). The nucleus of the atom, embedded in the near field of the coherently oscillating electron cloud (Biedenharn, Baldwin, et al., 1986), would generally experience a larger dipole field than that of the driving laser (Solem and Biedenharn, 1988). Furthermore, nonlinearity of the oscillating cloud would produce both spatial and temporal harmonics, so nuclear transitions of higher multipolarity could be driven, and they could also be driven at multiples of the laser's quantum energy (Solem and Biedenharn, 1987). A central theoretical result was that

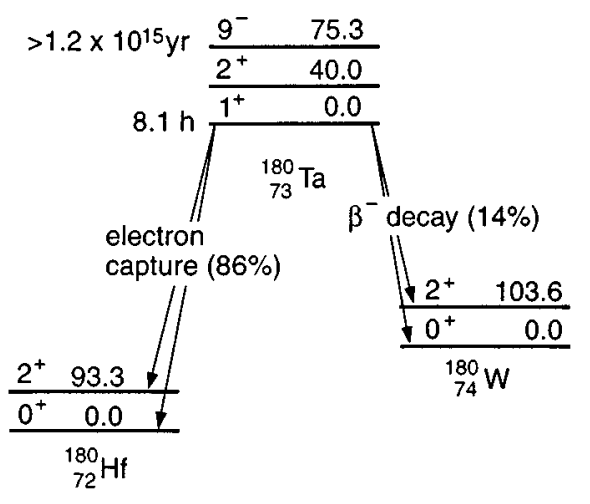

FIG. 11. Energy levels of three adjacent mass-180 isobars, showing the isomeric 9- state of ${ }^{180} \mathrm{Ta}$. According to Collins et al. (1995), a broad absorption band positioned above the 9-, can be excited by bremsstrahlung and deexcites the ${ }^{180} \mathrm{Ta}$ isomer to the 8.1-hour ground state.

$2^{2 n}$ poles could be driven only at even multiples of the laser frequency and $2^{2 n+1}$ poles at odd multiples, although the coupling became weaker as multipolarity and harmonic number increased.

Some researchers have challenged the Rhodes claim of coherent electronic oscillation, asserting that the data are equally well explained by conventional sequential ionization (Lambropoulos, 1985). Recent evidence for coherent electronic motion in atomic clusters (Boyer and Rhodes, 1985; Boyer et al., 1994; Thompson et al., 1994) at intensities near $10^{19} \mathrm{~W} \mathrm{~cm}^{-2}$ provides strong support for the Rhodes theory.

\section{b. Transfer by incoherent radiation}

Using bremsstrahlung from Linac sources, the Texas group has successfully accomplished interlevel transfer in several nuclides, in particular, the deexcitation of the naturally occurring isomer ${ }^{180} \mathrm{Ta}$ (Sec. VI.D.2), although, from Fig. 11, this requires an angular momentum change of eight units! This and other nuclides that they have investigated are highly deformed, with level schemes analogous to those giving rise to band spectra of molecules. In addition to the nuclear angular momentum, specified by the quantum number $I$, an additional quantum number $K$, measuring the projection of $I$ onto the nuclear body-fixed axis of elongation, is required to characterize them. For each value of $K$ there exists a set of levels $E(K, I>K)$, among which transitions can occur in accordance with certain selection rules. The particleplus-rotor model gives reasonable values for electric quadrupole transition probabilities with the same value of $K$ (Ring and Schuck, 1980). The collective part vanishes for transitions between different $K$, and such transitions are very weak because the single-particle part contains only the single particle matrix element. However, transitions between different $K$ are not strictly forbidden, and at high enough values of $K$, it has been long speculated that mixing of different $K$ states could occur.

Collins et al. have found that, in many nuclides, there is a fairly narrow band of excitation energies where 


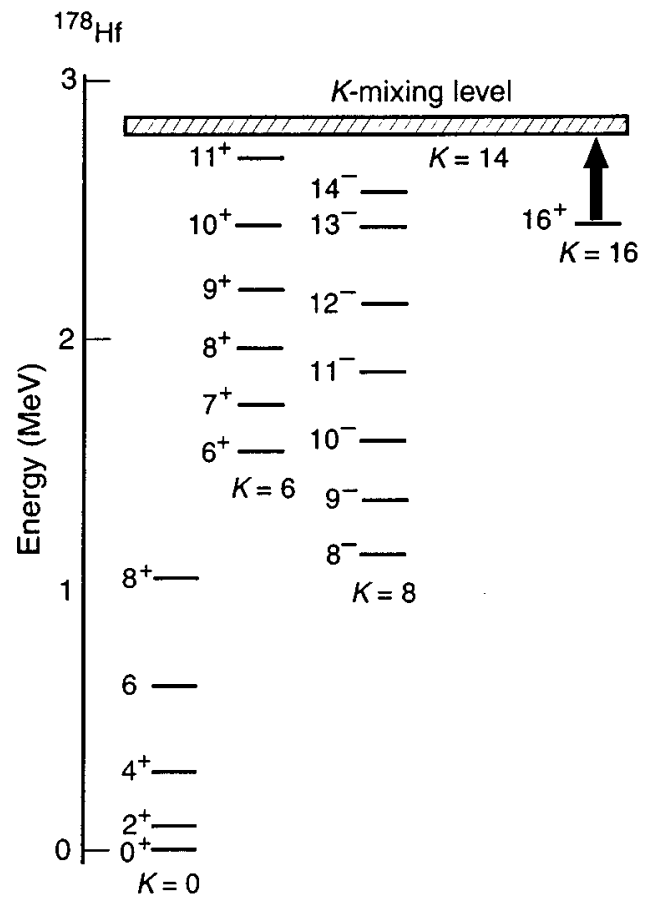

FIG. 12. Partial level scheme of the ${ }^{178} \mathrm{Hf}$ nucleus, showing the 31 -year $(K=16)$ isomer being excited to the broad $K$-mixing level. Two decay routes are shown: (1) a cascade in the $K=6$ levels to the $6^{+}$state followed by radiative emission to the 6 $+(K=0)$ with $922 \mathrm{keV}$ or to the $4^{+}(K=0)$ with $1247 \mathrm{keV}$, or (2) transition from the band $(K=14)$ to the $16^{+}(K=8)$ state followed by radiative emission to the $13^{-}$state with $140 \mathrm{keV}$ or to the $12^{-}$state with $437 \mathrm{keV}$.

these sets overlap, and so large changes of $K$ can occur. Thus an isomer such as the $I=9^{-}$state of ${ }^{180} \mathrm{Ta}$, for which transition to the $I=1^{+}$ground state is highly forbidden, can be "dumped" to the ground state by first exciting the nucleus with radiation to a higher " $K$-mixing" level, from which a chain of dipole transitions carries away the eight units of angular momentum. In the case of ${ }^{178} \mathrm{Hf}$ shown in Fig. 12 [Fig. 6 of Oganessian and Karamian (1995)], the 31-year isomer (I $=16^{+}, K=16$ ) can be transferred into the $K=6$ band or the $K=14$ band, in either of which cascades can occur leading to the $(0,0)$ ground state. Certain transitions are identified in the figure that, because of their measurable lifetimes, enable one to verify that transfer has taken place after a pulse of bremsstrahlung has been delivered. Many nuclides in the mass-island 150-200 associated with the "magic numbers" $(50,82,126)$ have been identified for which the integrated cross section $\sigma_{\gamma}$ is in the range 1 to $4 \times 10^{-26} \mathrm{~cm}^{2} \mathrm{keV}$. This value determines what will be required of a bremsstrahlung or radiative pump of narrower bandwidth.

\section{c. Preparing the storage isomer}

The essential first step in all proposals for indirect pumping from a storage state, namely, the generation and isolation of a pure isomer from other reaction products, requires an isomer-specific separation of nuclei in the storage state. A recoil ("Szilard-Chalmers") separation (Baldwin, Neissel, and Tonks, 1966) alone does not fully isolate the isomer from other reaction products. Characteristics that might be exploited to this end are nuclear mass and angular momentum. The mass effect is too small to be a practicable basis for a separation procedure. Because of the distinct nuclear spins of nuclear excited states, they could be separated in an atomicbeam apparatus, and there are isomer effects in hyperfine spectra (Melissinos and Davis, 1959) that can occasionally be exploited.

It has been proposed (Baldwin et al., 1978) to "tag" atoms containing the nuclear isomer by exciting them with optical laser radiation, sharply tuned to a selected hyperfine component of a resonance line. The selectively excited atoms can be scavenged with a chemical reagent or photoionized with a second laser and the ions collected with an electric field (Dyer and Baldwin, 1982). This principle was demonstrated in the case of ${ }^{198 m} \mathrm{Hg}$, using three-step photoionization (Dyer et al., 1983, 1986), although an isolated sample was not obtained (Sec. VI.E). In general, one must identify the lasant nuclide before one can devise a successful separation procedure-or even assume its feasibility-since this process depends critically on fine details of atomic spectra.

\section{d. Candidate nuclides}

Theoretical modeling (Arthur, Strottman, Arthur, and Madland, 1986) and searches of nuclear databases (Arthur and George, 1988) and the nuclear library (Artna-Cohen, 1988) have found no example of a longlived storage isomer separated by only a few $\mathrm{eV}$ from a short-lived state that could be the upper state of a grasing transition. The closest pairs of nuclear levels known are in ${ }^{229} \mathrm{Th}(4 \mathrm{eV})$ and ${ }^{235} \mathrm{U}(75 \mathrm{eV})$; neither is a graser candidate. Shape isomers have been suggested as the most likely category that can meet the nuclear requirements (Weiss, 1988).

The Texas scheme (Collins and Carroll, 1995) can use isomers of much higher energies, for example, ${ }^{178 m} \mathrm{Hf}$ $\left(E=2.447 \mathrm{MeV} ; T=31 \mathrm{y} ; I=K=16^{+}\right)$. This and many similar isomers have up until now been ruled out as candidates for graser application because of their very high excitation energies. Collins et al. dismiss this reasoning as "theoretical dogma."

The pumping scheme they have proposed for this isomer, from an extensive series of systematic studies with similar nuclei (Sec. VI.D.1), is shown in the energy-level diagram of Fig. 13. They estimate (Collins and Carroll, $1995)$ that irradiating ${ }^{178} \mathrm{Hf}$ with a short $(\sim 30$-ns) burst of $0.7-0.8-\mathrm{MeV}$ bremsstrahlung would reach the "giant $K$-mixing" level, leading to a cascade preferentially via the $K=6$ band, which would completely dump the stored energy. The final (93-keV, $1.5-\mathrm{ns}, 2^{+} \rightarrow 0^{+}$) transition would be low enough in energy for recoilless emission, provided that effects (recoil, ionization) of suddenly (78 ns) releasing $2.8 \mathrm{MeV}$, as well as the nonresonant part of the bremsstrahlung pump radiation, are nondestructive. 


\section{e. Difficulties}

Two major problems faced by all nuclear interlevel transfer schemes are (1) the angular momentum barrier and (2) survival of Mössbauer/Borrmann conditions.

Coherent optical transfer radiation, by multiphoton absorption, could supply the differences in energy and angular momentum between storage and upper lasant states, and if delivered into a superlattice (Sec. V.B.1), as in the multiwave Borrmann modes, would enable undoped regions of the host to provide heat sinks. However, no nuclide has been found that lends itself to this approach.

Collins et al. (1995) have shown how bremsstrahlung, with energy of the order of perhaps $30 \mathrm{keV}$ can overcome the angular momentum barrier (Sec. V.B.2) in isomers with energy in the $\mathrm{MeV}$ range. However, it is not certain that Mössbauer conditions can be preserved with a radiative pump, even if its bandwidth can be comparable with the as yet unknown width of the $K$-mixing resonance.

Collins et al. $(1992,1995)$ describe a hypothetical scenario in which a diamond host $0.67 \mu \mathrm{m}$ thick, doped to $10 \%$ with an isomeric parent for a $100-\mathrm{keV}$ lasing transition, is pumped to the $K$-mixing level by absorption of 30-keV bremsstrahlung. Collins and Carroll assume that (1) the resonant transfer pump band is $1 \mathrm{eV}$ wide; (2) the Borrmann effect reduces nonresonant absorption tenfold; (3) " ... edge filters or ablation layers could reduce the bandwidth of $30-\mathrm{keV}$ pump radiation to 3 keV ..."; (4) only 10-20\% of the primary photoelectrons would be stopped in the host film. With these extremely optimistic parameters, they estimate that an energy fluence $0.177 \mathrm{~J} \mathrm{~cm}^{-2} \mathrm{eV}^{-1}$ within the resonant bandwidth should suffice for gain, and conclude that the resultant temperature rise would not exceed the $2250-\mathrm{K}$ Debye temperature of the host.

We believe these conditions are unrealistic. At such a high degree of doping, the Borrmann effect could not occur; the effective Debye temperature would be only $590 \mathrm{~K}$ [Eq. (36)]; the mechanical shock from delivery of a multijoule transfer pulse cannot be neglected, nor can the recoils from the $2-\mathrm{MeV}$ gamma-ray cascade to the lasing level.

\section{Proposals for stimulating long-lived transitions}

\section{Line narrowing}

Inhomogeneous broadening, in particular, magnetic dipole-dipole and inhomogeneous isomer shift, eliminate many otherwise promising candidate nuclide/host systems from consideration, particularly those with transition lifetimes exceeding a few microseconds. Throughout the 1970s, concepts appeared (Ilinskii and Khokhlov, 1973; Gol'danskii et al., 1974) for line narrowing by NMR techniques in separated long-lived isomers. Ilinskii and Khokhlov (1973), and related proposals, used RF pulses and a static magnetic field to set the nuclear magnetic moments into rotation. The frequency shift of their $\gamma$-ray transitions, owing to interaction with magnetic moments of the host lattice, was therefore modulated by the rotation frequency. If the rotation period was short compared to the nuclear lifetime, the broadened line would break up into a comb of narrow lines resembling a frequency modulation spectrum. The carrier amplitude would be large compared to the sidebands, thereby effectively removing the broadening owing to interaction of the nuclear magnetic moment with its environment. This technique is similar to "motionnarrowing" methods used to resolve NMR lines, whereby tumbling of a molecule in a liquid can be used to narrow the NMR line of one of its constituent nuclei or mechanical rotation can be used for NMR narrowing in a powder.

Gol'danskii et al. (1974) showed that monopole broadening can be canceled by taking advantage of the fact that, for certain atomic species, both monopole shift and hyperfine splitting of nuclear states vary in proportion to the density of electrons at the nucleus. If the upper and lower states both suffered monopole broadening, it would not be possible to cancel them simultaneously.

These proposals were reviewed in depth in Sec. V.5. of Baldwin et al. (1981), where it was pointed out that, because of time-energy complementarity, inversion would be lost by spontaneous decay during the time required to reduce the linewidth sufficiently ${ }^{2}$ - an objection that would not apply to a system that did not require a population inversion.

\section{Inversion from radioactive decay}

One of the oldest ideas for creating nuclear population inversion (Baldwin, Neissel, Terhune, and Tonks, 1963a, 1963b) would use a transition from a long-lived upper state to a short-lived lower state. Having excited the long-lived upper state, one would simply wait for lower-state decay to create the inversion. This attractive possibility is defeated by homogeneous broadening - the natural linewidth, largely the uncertainty width of the lower state, greatly exceeds the radiation width of the upper state (Vali and Vali, 1963b).

Two decades ago this idea was revived (Kamenov and Bonchev, 1975; Greaves et al., 1987; Kamenov, 1988; Skorobogatov, 1990). Kamenov (1988), asserting that "homogeneous broadening does not exist," derived a formula for the stimulation cross section. His analysis, based on a modified Einstein coefficient, treats a threelevel system in thermodynamic equilibrium, in which thermal radiation induces transitions only between the higher pair of excited states, neglecting the induced transitions between the ground and first excited states- the transitions that broaden the lower excited state, homogeneously broaden the gamma-ray line, and, in fact, ac-

\footnotetext{
${ }^{2}$ To circumvent this difficulty, Balko and his colleagues proposed to maintain inversion by sustained thermal-neutron pumping, while simultaneously using the NMR line-narrowing techniques (Balko, 1988), but this requires an unattainably high flux of thermal neutrons.
} 
count for practically all of its natural breadth (see: Fig. 1). Inclusion of those transitions makes the stimulation cross section so small that lasing would be utterly impossible, despite the automatically assured population inversion (Baldwin and Solem, 1994).

Nevertheless, Kamenov (1988) proposes a specific design for a graser, namely, a long cylinder of ${ }^{124} \mathrm{Te}$ in a Be host lattice. It would be pumped by a thermal-neutron flux of $10^{13} \mathrm{~cm}^{-2} \mathrm{~s}^{-1}$, to activate ${ }^{125 m} \mathrm{Te}$. Kamenov supports his proposal by citing claims that stimulated emission has been observed in ${ }^{125 m} \mathrm{Te}$ (Alpatov et al., 1986; Skorobogatov and Dzevitskii, 1995; Kamenov, Christoskov, Petrakiev, and Kamenov, 1995). Those experiments and their significance are discussed in Sec. VI.C.2.

\section{Homogeneous broadening by an NMR technique}

Rather than considering it inimical to lasing, Boolchand and Coussement (1988) proposed to exploit homogeneous broadening, using NMR level-crossing techniques to increase the homogeneous linewidth until the various inhomogeneously displaced nuclear resonances overlap, making the homogeneous breadth dominant. Later they withdrew the proposal.

\section{Subinversion lasing: Proposals for suppressing resonant absorption}

Population inversion is required for stimulated transitions to outnumber resonant absorptions. That requirement is not absolute if one can suppress resonant absorption. Lasing without inversion has actually been accomplished in optical laser research (Scully and Fleischauer, 1994), by techniques that use destructive interference (Kocharovskaya and Kanin, 1988; Harris, 1989; Scully and Zhu, 1990; Zibrov et al., 1996), establishing opposite phases to two substates of one of the lasing levels by means of a fourth level (" $\Lambda$ " or "P" schemes). How, even whether, those techniques can be applicable to nuclear transitions remains to be demonstrated. Because of nonresonant absorption, the excitation requirement is still not negligible, although, in the absence of Borrmann mode formation, it can be reduced by only about three orders of magnitude, five with Borrmann modes.

In principle, nuclear transitions offer other possibilities for lasing without inversion, namely, recoil shift in unbound nuclei (Marcuse, 1963) and techniques based on NMR.

\section{An optically pumped traveling-wave amplifier}

Vysotskii and Vorontsov (1977) have proposed a parametric amplifier for recoilless radiation, comprising an optically transparent medium doped with excited nuclei and supporting an optical laser transition. The optical, but not necessarily the nuclear, states are population inverted by an appropriate pump. The signal wave to be amplified by a three-wave interaction is recoilless gamma radiation, introduced at one end; stimulated optical transitions provide the idler wave; the third wave is supplied by that part of the Doppler-broadened gamma radiation that satisfies phase-matching and frequency conditions. We are aware of no further work on this proposal.

\section{Exploiting selection rules in a polarized population}

Vysotskii (1978) has pointed out that, if the upperstate nuclei could all be put into the substate $m_{u}=I_{u}$, while simultaneously all lower-state nuclei were in the substate $m_{l}=I_{l}$, then the selection rules for the magnetic quantum number would give an effective population inversion. The difficulty with this operation is that the time required for optical or microwave pumping to polarize the upper states would greatly exceed the lifetimes of states that can emit a narrow recoilless line. Vysotskii (1978) suggests polarizing the ground states using a strong internal magnetic field and very low temperature, and pumping radiatively to obtain partially polarized upper states. Selection rules would then permit emission to empty lower-state sublevels. He gives a numerical example for the Mössbauer isomer ${ }^{161} \mathrm{Dy}$. Although this procedure would be an exception to the general rule that radiative pumping of a two-state system cannot create a population inversion, the required intensity and bandwidth of the pump would be obtainable only from another graser using the same transition!

\section{Using RF to induce hyperfine transitions}

An analogy to the optical inversionless technique might oppositely phase a pair of Zeeman sublevels, using RF to induce transitions to a third sublevel; unfortunately, selection rules preclude this, and it has been shown that mixing two levels with radio frequency cannot completely eliminate resonance absorption (Fill et al., 1992).

\section{Level-crossing concept}

Coussement et al. $(1993,1988,1995)$ have proposed that a level-crossing technique could in principle make the four-level approach possible using coherent radiation sources of accessible quantum energy. In their idealized case, as illustrated in Fig. 13, lower states with spin $I_{l}=3 / 2$ and upper states with spin $I_{u}=1 / 2$ reside in a noncubic uniaxial crystal. The nuclear quadrupole interaction with the crystal's electric-field gradient splits the lower level into the doublet $| \pm 1 / 2\rangle$ and $| \pm 3 / 2\rangle$. A magnetic field is applied parallel to the axis.

The magnetic field splits the lower states into four Zeeman levels, $|1 / 2\rangle,|-1 / 2\rangle,|3 / 2\rangle$, and $|-3 / 2\rangle$, while the upper state is split into two Zeeman levels, $|1 / 2\rangle$ and $|-1 / 2\rangle$. The $|-3 / 2\rangle$ level makes two crossings as the field is increased, first with the $|1 / 2\rangle$ level, then with the $|-1 / 2\rangle$ level. It is near this second crossing that suppression of resonant absorption can be realized.

If the magnetic field is slightly misaligned with the axis of the crystal's electric-field gradient, the Zeeman levels mix, forming the doublet $|1\rangle=(|-1 / 2\rangle-|-3 / 2\rangle) / \sqrt{2}$ and $|2\rangle=(|-1 / 2\rangle+|-3 / 2\rangle) / \sqrt{2}$. The energy spacing of the 


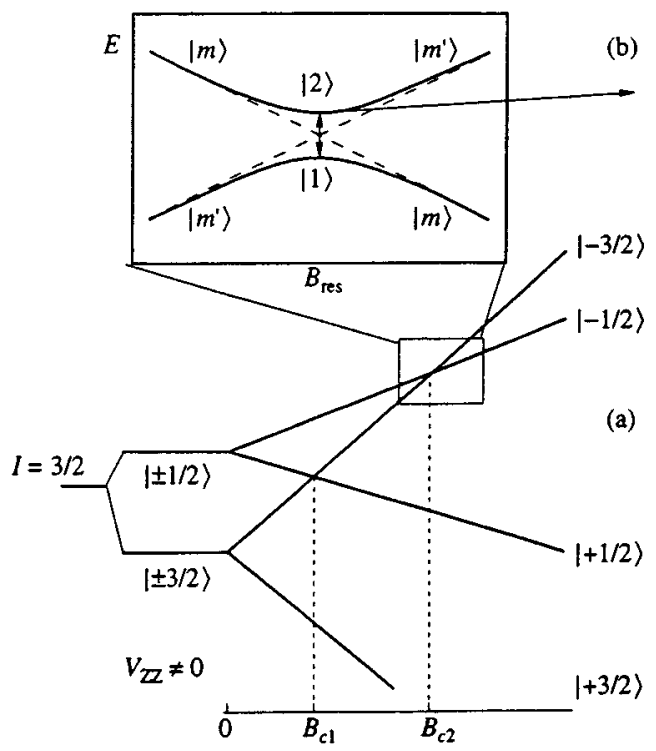

FIG. 13. Level mixing according to Coussement (1995): (a) hyperfine levels for a nucleus submitted to an axially symmetric electric-field gradient and a magnetic field; (b) if the electric-field gradient is at an angle with respect to the magnetic field, the hyperfine levels are mixed near a level crossing, the level populations are equalized, and the degeneracy is lifted. Figure courtesy of R. Coussement.

doublet can be adjusted to ensure that it is less than the sum of the radiative and homogeneous widths of the nuclear transition. Destructive interference of the mixed levels can then be driven from the $|1 / 2\rangle$ Zeeman level of the lower nuclear state.

Because the mixed states and the $|1 / 2\rangle$ level are Zeeman levels of the same nuclear state, the energy spacing can be fairly small, probably in the microwave regime. With the microwave field on, resonant gamma-ray absorption from the doublet $|1\rangle$ and $|2\rangle$ of $I_{l}$ and the Zeeman level $|-1 / 2\rangle$ of $I_{u}$ should be totally suppressed. If no other absorption mechanisms were in play, gammaray lasing should be realized with any excitation of $I_{u}$.

The Coussement scheme seems very promising and may stimulate researchers to find an appropriate nuclide and host to put the concept into practice. It may also inspire other ideas for suppressing resonant absorption while preserving the Mössbauer effect. Of course, decay during the time required to create the altered level scheme must be considered in examining the kinetics of lasing in such a system.

Any schemes that use external radiation for phasing the lower states must avoid the graser dilemma.

\section{E. Miscellaneous concepts}

Several concepts have entered the literature recently that fit none of the general categories reviewed above.

\section{Three-boson stimulated scattering}

Skorobogatov (1980) proposed producing a so-farunobserved phenomenon-three-boson stimulated scattering-occurring during intersection at definite angles of three laser beams. (Also see Skorobogatov and Dzevitskii, 1973); Skorobogatov, 1975. He suggests that the high flux intensities already achieved at wavelengths 100-1720 $\AA$ and at $1 \mathrm{~cm}$ should, in principle, make feasible a frequency doubler with an efficiency of order $100 \%$ and, with a cascade of frequency doublers, a gamma-ray laser.

\section{Nuclear Raman effect}

On the basis of available data, Ishkanov and Piskarev (1980) analyzed the use of the nuclear Raman effect for pumping a gamma laser and showed that available electronic accelerators might provide the necessary concentration of excited nuclei. They did not show how the concentration would be achieved without destroying the conditions necessary for substantial gain.

\section{Rapid heat removal under high pressure}

Winterberg (1986) suggested that the graser dilemma could be circumvented by rapid heat removal under high pressure and that the effects of heat produced during pumping could be defeated by enhancing electronic thermal conductivity under high pressure. While the physical effect is qualitatively correct, we find that the augmented heat transfer is insufficient.

\section{Quasi-superradiance}

Chaplin and Xu (1992; see also Xu, 1992) have an interesting concept, which they call "nuclear quasisuperradiance," or "QSR." A relatively long-lived radionuclide in a solid host decays (beta, electron capture, or isomeric transition) to a short-lived lowest excited state of a daughter nuclide, which emits recoilless gamma radiation in transition to the ground state. They argue that, if a reference direction has been established in the solid by nuclear orientation in a magnetic field, resonance absorption of the recoilless gamma rays should cause the Dicke inversion number (Dicke, 1954) $m$ to change less rapidly than in ordinary decay and establish partial coherence in the gamma emission. $\mathrm{Xu}$ claimed to have observed a small effect of this nature in ${ }^{54}$ Cr. For comments, see Sec. VI.C.3.

\section{EXPERIMENTAL WORK}

Graser research is no longer purely theoretical and speculative. Recently, relevant experiments have observed recoilless emission in long-lived transitions, have studied the transmission of radiation in resonant media, may have observed coherent gamma emission, and have investigated problems of two-step pumping, viz, isomer separation and interlevel transfer. 


\section{A. Mössbauer effect in ${ }^{109} \mathrm{Ag}$}

Attempts have often been made to observe the Mössbauer effect in nuclei of lifetimes much longer than those typical of Mössbauer spectroscopy, particularly ${ }^{109} \mathrm{Ag}, T=40 \mathrm{~s}$. The $88-\mathrm{keV}$ gamma ray that follows beta decay of the ${ }^{109} \mathrm{Cd}$ parent should have an appreciable recoilless fraction $(0.7 \%$ at $77 \mathrm{~K}, 5 \%$ at $4.2 \mathrm{~K})$ in silver, but the natural linewidth, $1.15 \times 10^{-17} \mathrm{eV}$, is small-comparable with gravitational redshift between parts of the same sample-and Mössbauer's Dopplershift technique cannot be used.

Bizina et al. (1963) employed a resonance activation technique: they irradiated a silver foil to create ${ }^{109} \mathrm{Cd}$, annealed and then exposed it in a cryostat to another, similar foil. The latter was then removed and counted to measure any activity resulting from resonance absorption of gamma radiation from the first foil. Alpatov et al. (1977) modified the experiment to prevent mechanical transfer of activity when the foils were in contact.

Wildner and Gonser (1979) used a self-absorption technique, in which decay of the ${ }^{109} \mathrm{Cd}$ parent in a single foil was counted. They observed a change in the $88-\mathrm{keV}$

${ }^{109} \mathrm{Ag}$ gamma-ray activity when the foil's temperature was changed. They attributed the residual shift, after correcting for thermal contraction, to temperature dependence of the recoilless fraction with attendant change in self-absorption.

Hoy and Taylor (1988) improved this technique, simultaneously counting the gamma rays and the $\mathrm{x}$ radiation associated with their internal conversion. The change in self-absorption due only to change in the recoilless fraction could be inferred from the ratio of these data as a function of temperature. Their latest experimental curves (Reszaie-Seraj et al., 1995), shown in Fig. 14, show a small Mössbauer effect at the lowest temperature. An attempt to observe gravitational broadening was not successful.

There does indeed seem to be an observable Mössbauer effect in these silver isotopes, indicative of less broadening than one might expect. The large spread in the data from these experiments (Table IV) is probably from differences in the silver samples. None has attempted to determine the line shape. This might be done by measuring the time dependence of the transmitted radiation (Baldwin, 1979; Baldwin, Solem, and Gol'danskii, 1981; see also Sec. II.B.4).

The measured recoilless fraction is inadequate for net stimulated-emission gain, and, even if it were high enough, the kinetics of a graser based on such a slow transition makes it a doubtful objective.

\section{B. Time filtering in crystals}

A classic experiment by Lynch et al. (1960), on "time filtering" of resonantly transmitted recoilless radiation, observed the time dependence that is described by a function similar to that derived in Sec. II.B.3, Eq. (18).

Smirnov et al. (1984) studied the time dependence of pulsed polarized ${ }^{57} \mathrm{Fe}$ Mössbauer radiation resonantly
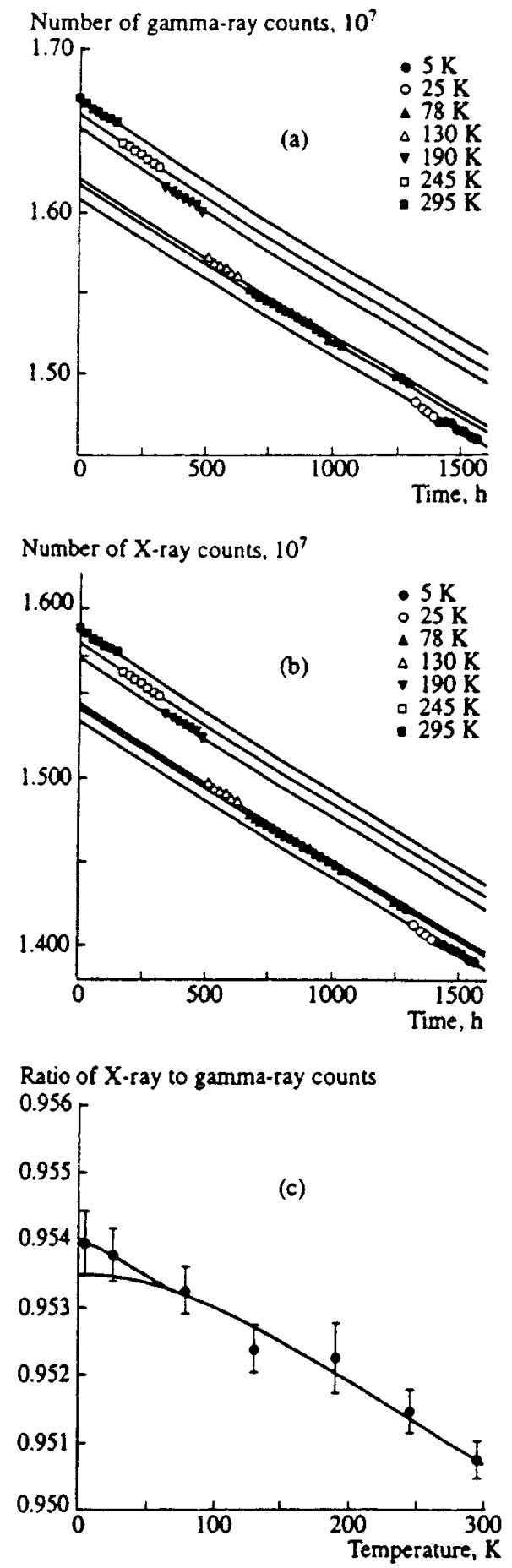

FIG. 14. Counting rates of (a) gamma rays and (b) x rays from ${ }^{109} \mathrm{Ag} / \mathrm{Ag}$ as functions of time and temperature, and (c) their ratios as a function of temperature, measured by Rezaie-Serej et al. The lower curve is that expected if there were no Mössbauer effect. From Rezaie-Serej et al. (1995). Figure courtesy of G. Hoy.

scattered in a single crystal and found that the nuclear excitation decayed much more rapidly when it was collectively, rather than incoherently, excited. The source radiation was passed through three identical thin crystals of antiferromagnetic ${ }^{57} \mathrm{FeBO}_{3}$ : the first for selecting one hyperfine ( $\pi$-polarized) component, the second a mag- 
TABLE IV. Measured Mössbauer Effect in ${ }^{109} \mathrm{Ag} / \mathrm{Ag}$.

\begin{tabular}{lcc}
\hline \hline Reference & Method & $\mathrm{f} / \mathrm{f}_{0}$ \\
\hline Bizinia et al., 1963 & Activation transfer & $3 \times 10^{-6}$ \\
Alpatov et al., 1977 & Activation transfer & $?$ \\
Wildner and Gonser, 1979 & Self-absorption vs $T$ & $10^{-2}$ \\
Rezaie-Serej et al., 1995 & Self-absorption vs $T$ & $1.0 \pm 0.2 \times 10^{-2}$ \\
\hline \hline
\end{tabular}

netic shutter with which they could determine the arrival time of the square pulse of radiation at the third crystal, and the third as the resonant scatterer. They found that the intensity of the scattered radiation increased slowly after an initial delay, but that the rise time was much shorter when the crystal and detector were oriented for Bragg (coherent) scattering than at other angles, when the scattering was incoherent.

Such experiments are handicapped by the limited intensity of radioactive sources. Synchrotron radiation, although very intense, must be filtered to isolate the resonant radiation, leaving also a low intensity, but with the advantages of short pulse duration, excellent collimation, and intrinsic polarization, Many experiments (Rüffer et al., 1987; Van Bürck et al., 1987, 1992) have demonstrated-in addition to accelerated decay of the collective nuclear excitation when the Bragg condition is satisfied-beats between hyperfine components of the resonantly scattered radiation, total reflection from the crystal face in Bragg geometry, and anomalous transmission (Borrmann effect) in the Laue geometry. In the last case, the intensity and the angular and energy distributions of the transmitted radiation demonstrate that in the case of "collective" nuclear scattering the amplitude for forming the excited nuclear state vanishes (Kagan and Afanas'ev, 1964) so that there is no absorption due to other radiative modes of nuclear decay (e.g., internal conversion).

In all those experiments, the source of the radiation "is external to the crystal." It is desirable to verify the material of Sec. II.E, in which the radiation originates from, is scattered by, and stimulates emission from nuclei "within the crystal."

To avoid differences between source and filter environments, Hoy and his collaborators (Hoy and Taylor, 1988) doped natural-Fe single crystals on one face with parent ${ }^{57} \mathrm{Co}$ and measured decay curves of the $14.4-\mathrm{keV}$ gamma rays from ${ }^{57 m} \mathrm{Fe}$ emerging from opposite faces of the iron crystals, using the delayed-coincidence technique employed for "time-filtering" experiments (Lynch et al., 1960). Radiation from the doped face decayed exponentially; the transmitted radiation, nonexponentially. A rapidly decaying "prompt peak" was followed by additional time structure. Resonant absorption and delayed reemission of the gamma radiation redistributes it in time.

Hoy's group has begun an investigation of the anomalous emission and transmission of gamma radiation from internal sources in single crystals, intended ultimately to confirm the existence of the higher-order wave modes predicted by Hutton et al. (discussed in Sec. II.E.2). The anomalous transmission of externally incident $\mathrm{x}$ radiation has been investigated thoroughly (Batterman and Cole, 1961), but there has been no work in which the sources are within the crystal.

\section{C. ${ }^{125} \mathrm{Te}$ experiments}

Population inversion is automatic, but the resonance cross section is small when the lower laser state has a much shorter lifetime than the upper. Bonchev and Kamenov (1975) propose to make a graser using ${ }^{125} \mathrm{Te}$, dispute the usual objection that the cross section for stimulated emission of the $109-\mathrm{keV}$ gamma ray cannot exceed $1.2 \times 10^{-42} \mathrm{~cm}^{-2}$, and claim that stimulated emission of the $109.27-\mathrm{keV}$ gamma ray has actually been observed as ${ }^{125} \mathrm{Te}$ decays by an M4 transition to the short-lived lower state.

\section{Alpatov's experiments}

Alpatov et al. (1986) measured the gamma-ray spectrum with a pulse-height analyzer and found, besides the expected strong peak for $109 \mathrm{keV}$ a small peak in channels that correspond to $218 \mathrm{keV}$. Such a "doubleenergy" peak is the result of simultaneous absorption of two 109-keV photons within the resolving time of the detector; they attributed it to coherent pairs of photons from stimulated emission. Because accidental coincidence could account for the claimed effect (their paper gave no data for counting rates and resolving time), their claim was disputed (Solem and Baldwin, 1995).

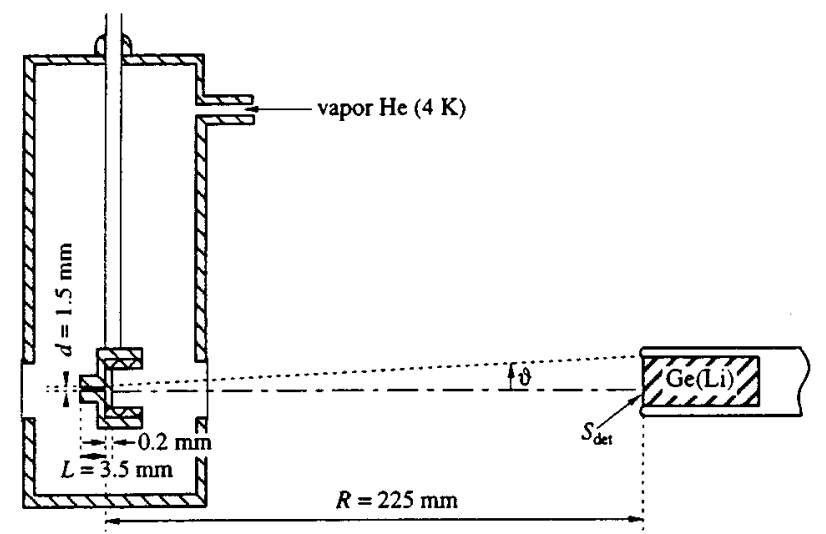

FIG. 15. Skorobogatov's apparatus for the ${ }^{125 m}$ Te measurements. From Skorobogatov and Dezevetskii (1995). Figure courtesy of G. Skorobogatov. 


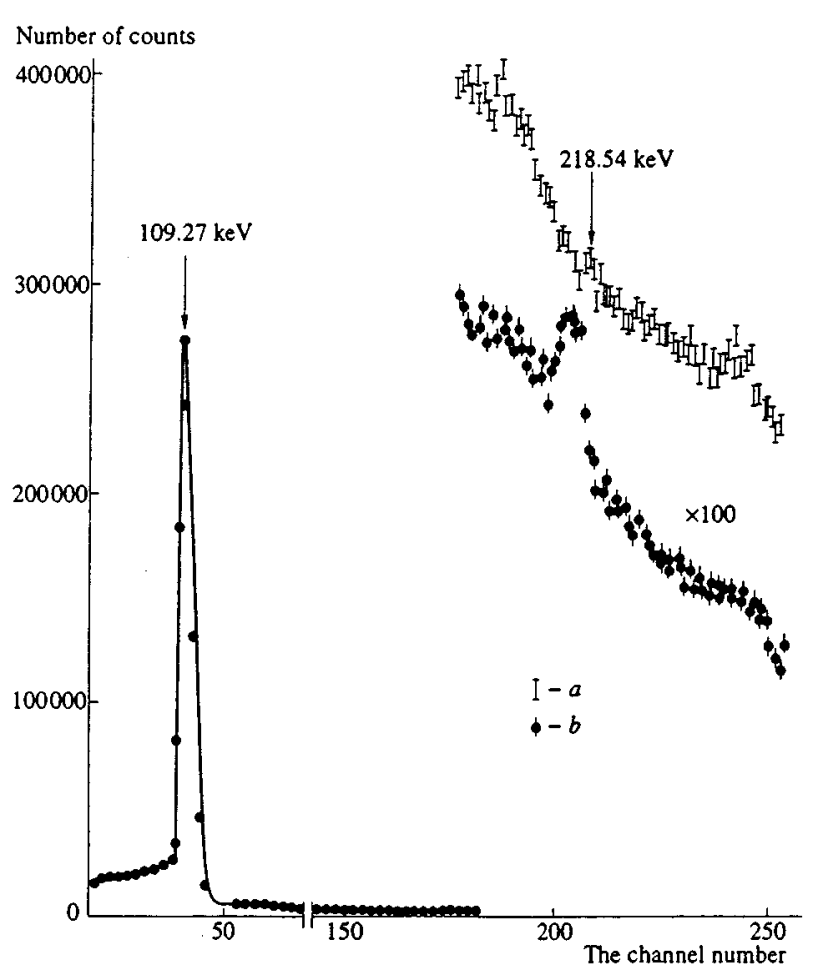

FIG. 16. Gamma-ray spectra from the ${ }^{125 m} \mathrm{Te} / \mathrm{Be}$ sample at (a) $300 \mathrm{~K}$ and (b) $10 \mathrm{~K}$. From Skorobogatov and Dzevitskii (1995). Figure courtesy of G. Skorobogatov.

\section{Skorobogatov's experiments}

Recent work by Skorobogatov and Dzevitskii (1995) on both Te isomers cannot be so dismissed. The count in the 218-keV channel is appreciable only when the sample is cooled to $10 \mathrm{~K}$ (Figs. 15, 16). We find it difficult to account for the absence of accidental coincidences in the room-temperature case. With any reasonable assumption about resolving time at the counting rate reported for the $109-\mathrm{keV}$ gamma rays, accidentals should have produced counts in the $218-\mathrm{keV}$ channel.

Assuming the effect was real, Skorobogatov and Dzevitskii deduced a cross section for creating the 218$\mathrm{keV}$ photons, which happens to agree, within experimental error, with the "wave cross section," rather than the far smaller value given by the accepted formula.

In their 1995 article Skorobogatov and Dzevitskii (1995) argue that, at the low temperatures where they observe the two-photon coincidences, the de Broglie wavelengths of the nuclei exceed both the internuclear spacing in BeTe and the gamma-ray wavelength. They state that, because, "Spatial synchronization occurs by the moving wave mechanism under superradiance of an extended system," each microcrystallite in their sample should be capable of coherent spontaneous emission, and deduce that the coherent component of the resultant emission will be a fraction.

$$
\left(1+\frac{N_{2}}{\mu^{3}}\right) \frac{f \beta \tau_{1}}{2(1+\alpha)\left(\tau_{2}+\tau_{1}\right)}
$$

of the incoherent component. This agrees within experimental error with the measured ratio of the "two- photon" to the single-photon counting rate. It appears ${ }^{3}$ that these experiments have detected weak nuclear superradiance (NSR). However, in these nuclides, the lower state is so short lived that an appreciable population cannot accumulate, so, as noted in Sec. II. B. 5, existing theories of NSR cannot explain how the coherence can develop. For example, the Dicke formula for the enhanced radiation rate, Eq. (19), was derived for a system in which the sum of upper- and lower-state populations is constant.

Even though this type of system cannot be further developed to create an actual laser, confirming the observation of coherent spontaneous emission (weak superradiance) from a nuclear isomer would be a significant milestone in this field. It is essential that this experiment be independently performed and extended, for example, to look for directional time, and temperature dependences, measure the two-photon linewidth, and find other cases.

\section{Xu's experiment}

$\mathrm{Xu}$ (1992) reports a "small positive effect" with the 834-keV gamma ray that accompanies decay of ${ }^{54} \mathrm{Mn}$ to ${ }^{54} \mathrm{Cr}$ from radiomanganese in a nickel host in a strong magnetic field at low temperature. He attributes this to quasi-superradiance (Sec. V. E. 4). We cannot accept this explanation. The recoil energy from emission of this gamma ray is so high that the recoilless fraction is utterly negligible; thus the reabsorption essential to QSR cannot occur.

\section{Interlevel transfer}

1. Search for multiphoton absorption in ${ }^{235} U$

As a test case for two-step pumping, Bounds and Dyer (1992) attempted to accomplish a laser-induced transfer between two nuclear states by multiphoton absorption. They irradiated ${ }^{235} \mathrm{U}\left(I=7 / 2^{+}\right)$with a $248-\mathrm{nm}$ $\mathrm{KrF}$ laser at power levels as high as $2.5 \times 10^{15} \mathrm{~W} \mathrm{~cm}^{-2}$ and looked for the low-energy conversion electrons 4 from decay of the $75-\mathrm{eV}$ isomer ${ }^{235 m} \mathrm{U}\left(I=1 / 2^{-}, T=25\right.$ $\mathrm{m})$.

In their apparatus (Fig. 17), uranium vapor, formed by laser heating on the end of an enriched-uranium wire, was exposed to pulses of the $\mathrm{KrF}$ laser radiation, forming ions that were collected on a target plate. After exposure, the collector plate was moved into an adjacent, evacuated chamber for counting the extremely-lowenergy conversion electrons from ${ }^{235 m} \mathrm{U}$ decay. A major difficulty was a background of electrons with comparable energies. From their data, Bounds and Dyer esti-

\footnotetext{
${ }^{3}$ Unfortunately, the reference cited by the authors, an untranslated Russian text, is unavailable to us.

${ }^{4}$ The energy of the isomeric state is uncertain by about $5 \mathrm{eV}$. Since approximately 15 times the 5-eV laser photon energy and three units of angular momentum must also be supplied, the chance for a successful experiment is admittedly very low.
} 


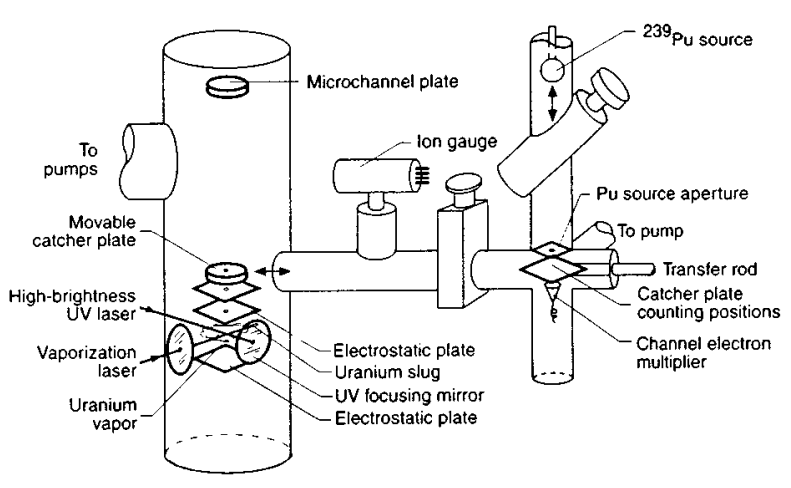

FIG. 17. Schematic of the ${ }^{235 m} \mathrm{U}$ interlevel transfer experiment of Bounds and Dyer (1992).

mated that the probability of nuclear excitation in ${ }^{235} \mathrm{U}$ at the maximum power level available is no greater than $4 \times 10^{-5}$ (Bounds and Dyer, 1992).

\section{The Texas experiments}

Collins and his collaborators (the review by Collins, Carroll, Oganessian, and Karamian 1995, contains references to earlier papers) report a large number of experiments in which they have deexcited nuclear isomers by bremsstrahlung generated in LINAC targets by electrons of energy $>2 \mathrm{MeV}$ (Anderson et al., 1988; Carroll et al., 1993), despite the generally high angular momentum change ( 8 units in the case of ${ }^{180 m} \mathrm{Ta}$ !) They show that this $\left(\gamma, \gamma^{\prime}\right)$ reaction has a "giant resonance," which decays to lower excited states that might serve as upper lasant states for grasers (Collins et al., 1993; see Fig. 11), and support their proposal for two-step pumping related in Sec. V.B.2. They list 29 nuclides that might be pumped to inversion by this reaction, the most promising of which are hafnium isotopes.

Carroll et al., (1991) and Collins et al. (1995) have shown that the effect is not caused by inelastic scattering of background photoneutrons produced by $(\gamma, n)$ reactions, but is due to photons, and the effect has been confirmed by other workers.

It is difficult to see how one can use this process to pump a two-step graser that would require both population inversion and recoilless transitions. For every nucleus that is pumped, there will be thousands of useless photons, creating heat and lattice damage-an extreme case of the graser dilemma.

This group has also conducted other investigations related to graser development, in particular, studies of novel ways to generate diamond-film hosts (Davanloo et al., 1990).

\section{E. Isomer separation and the ${ }^{197} \mathrm{Hg}$ experiments}

All two-step-pumping proposals assume that the active nuclide can be made available in a "storage" state; i.e., as a pure isomer. Separation can in principle be accomplished by on-line atomic-beam methods or by selectively exciting a hyperfine component of its optical spectrum with a laser or resonance lamp, followed by
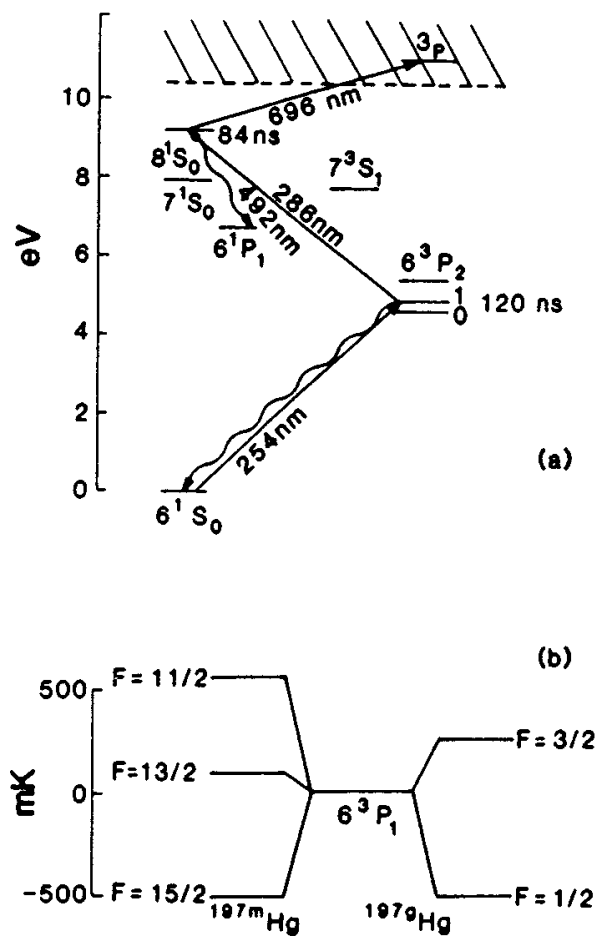

FIG. 18. Isomer separation experiments: (a) Energy levels of $\mathrm{Hg}$, showing the succession of excitation steps for isotopically or isomerically selective photoionization. (b) Hyperfine splitting of the two ${ }^{197} \mathrm{Hg}$ isomers. From Dyer et al. (1985a).

either chemically scavenging or photoionization (Secs. III.E and V.B.c). That, in practice, the latter may not always be possible is shown by the following experiment:

\section{Choice of isomer}

Dyer et al. (1985b) attempted to separate the two isomeric forms of ${ }^{197} \mathrm{Hg}$ (excited state, $299 \mathrm{keV}, I=13 / 2^{+}$, $T=24 \mathrm{~h}$; ground state, $I=1 / 2^{-}, T=64 \mathrm{~h}$ ) by three-stage selective photoionization, exploiting the hyperfine structure of this isotope as described by Melissinos and Davis (1959). This nuclide is not a candidate for graser application; rather, it was chosen because of its convenient spectroscopic, physical, and nuclear properties, viz., known atomic spectrum, high vapor pressure, high transition energy, relatively long half-lives, convenience in manipulation, and producibility in reasonable yield.

\section{Procedure}

After a series of preliminary experiments on selective photoionization of isotopes of natural $\mathrm{Hg}$ (Dyer et al., 1985a), ${ }^{197} \mathrm{Hg}$ was generated by bombarding a stack of thin gold foils with 16-MeV deuterons from a Van de Graaff accelerator at Los Alamos. Mercury that formed in the ${ }^{198} \mathrm{Au}(d, 2 n){ }^{197} \mathrm{Hg}$ reaction was distilled and collected on another gold foil, which was sealed in glass and flown promptly to the Laser Center at the Massachusetts Institute of Technology, where it was introduced into a glass cell for the separation experiments and heated to expel the radiomercury. Three collinear, tunable laser 


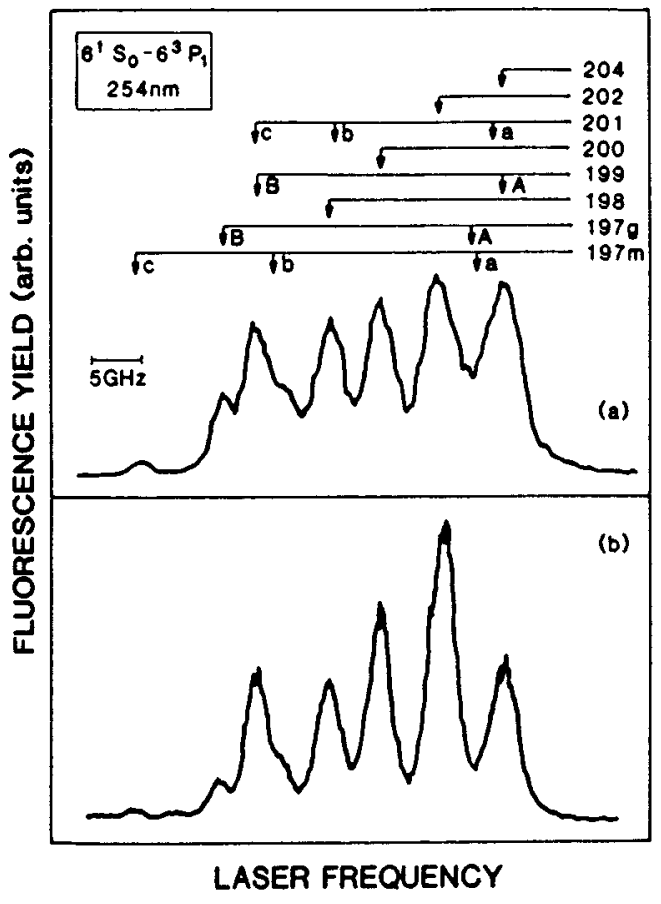

FIG. 19. Fluorescence excited by scanned by 254-nm radiation in $\mathrm{Hg}$ vapor from a deuteron-bombarded $\mathrm{Au}$ target. Scans (a) and (b) were taken several hours apart. From Dyer et al. (1985a).

beams were passed through a $10-\mathrm{cm}$ path in the $\mathrm{Hg}$ vapor between two parallel iron electrodes. Figure 18 shows the excitation steps:

$$
6^{1} S_{0} \stackrel{254 \mathrm{~nm}}{\Rightarrow} 6^{3} P_{1} \stackrel{286 \mathrm{~nm}}{\Rightarrow} 8^{1} S_{0} \stackrel{696 \mathrm{~nm}}{\Rightarrow}{ }^{3} P \stackrel{\text { autoionization }}{\Rightarrow} \mathrm{Hg}^{+} \text {. }
$$

Despite care in pretreatment of the gold foils, natural mercury was present. Scanning of the frequency of the 254-nm laser to excite fluorescence showed two additional lines of ${ }^{197} \mathrm{Hg}$, well separated from the five resolved components of the resonance line of natural mercury (Fig. 19). The two additional lines decayed at the expected rates. This laser was then tuned to one ${ }^{197} \mathrm{Hg}$ component, the 286-nm laser was scanned to identify its components and, finally, fixed on ${ }^{197} \mathrm{Hg}$-c; ions were collected for a long exposure, the electrodes were removed, and their various gamma-ray activities were measured.

\section{Results}

Figure 20, which shows ionization current as a function of the 286-nm scanning frequency when the 254-nm laser was tuned to the ${ }^{197 g} \mathrm{~B}$ line of Fig. 19, demonstrates that isomerically selective photoionization is feasible in this element. However, the measured electrode activities were much greater than expected from the charge collected and did not show enrichment in either ${ }^{197 m} \mathrm{Hg}$ or ${ }^{197 g} \mathrm{Hg}$. The excess activity was attributed to nonselective adsorption of active mercury on the electrode, and the failure to obtain an isomerically enriched sample outside the cell was ascribed to ion-neutral charge exchange.

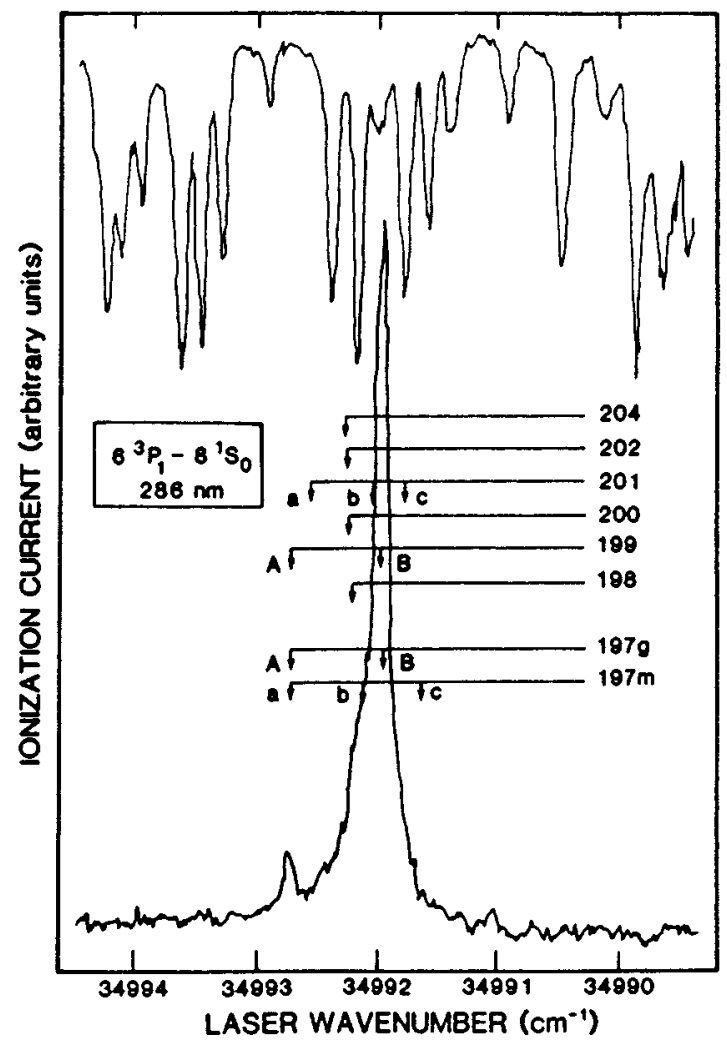

FIG. 20. Ionization current from $\mathrm{Hg}$ selectively excited by tuned lasers. The large peak is due to ${ }^{197 g} \mathrm{Hg}$. From Dyer et al. (1985b).

\section{Conclusions}

Instead of collecting ions, one might try chemical scavenging after only a single selective excitation step, not only with a tuned laser, but also with a ${ }^{204} \mathrm{Hg}$-enriched mercury lamp, after all traces of natural $\mathrm{Hg}$ in the gold foils had been removed. Mercury in the $6^{3} P_{1}$ state reacts more rapidly than $\mathrm{Hg}$ in its ground state with oxygen or water vapor (Baldwin et al., 1978).

In general, isomer separation involves major problems-of laser spectroscopy, radiochemistry, surface chemistry, and gaseous electronics-unique to each element and to each nuclide that might otherwise be an acceptable candidate for a graser.

\section{RECOMMENDATIONS}

\section{A. Present status}

Despite the extreme difficulty and interdisciplinary character of the graser field, the potentiality of nuclear sources of coherent ultrashort-wavelength radiation and the novel physics involved continue to attract workers from institutions in many countries. New ideas continue to appear and long-discarded ideas continue to be revived. The increasing number of experimental papers is a particularly encouraging sign that the field is no longer 
largely speculative, and the possibility that nuclear superradiance has at last been observed should encourage more work of that nature.

However, the graser dilemma continues to dominate the list of problems. In times when support for all research diminishes, those efforts that can still be made in this field should be in areas that promise success.

The following schemes seem unpromising:

(1) Direct pumping to inversion with neutrons.

(2) Concepts that feature decay-created inversion between nuclear states of lifetimes longer than several seconds.

(3) Stimulated transitions between states of lifetimes longer than a few seconds.

(4) Two-step schemes that feature transfer from a longlived storage state.

(5) Incoherent bremsstrahlung as either a primary pump (from a stable parent to a lasant state) or as a secondary pump (from a storage isomer).

The recent emergence of plausible schemes for eliminating resonance absorption offers a reduction by many orders of magnitude in the excitation requirement and so may at last resolve the lasing vs pumping dilemma, making it feasible to (1) use both recoilless transitions and multiwave modes in single-crystal resonators; (2) permit the use of line narrowing procedures that were previously ruled out by the time-bandwidth constraint. Still, this principle has yet to be proved applicable to nuclear transitions in general, and no specific nuclear system has yet been identified for which a practicable procedure would establish a resonant-absorption-free state. Moreover, although eliminating resonant absorption would greatly reduce the required pump intensity, nonresonant absorption would still impose a high threshold, so that both the Mössbauer and Borrmann effects would be essential.

It is too early to tell whether nuclear superradiance can be exploited to create coherent sources.

\section{B. Approaches that merit further investigation}

(1) Inversionless lasing; in particular, the Coussement proposal.

(2) Line-narrowing techniques-if and when inversionless lasing appears likely of realization.

(3) Recoil-based inversionless lasing.

(4) Nuclear superradiance.

\section{Approaches that lack promise}

(1) Inversionless lasing by the $\Lambda$ or $P$ schemes (Kocharovskaya, 1995).

(2) Two-step concepts that involve large $(\sim \mathrm{keV})$ transfer energy.

\section{Approaches in which we see no merit}

(1) Lasing from long-lived ( $T>$ several seconds) states.
(2) Direct pumping with neutrons of any energy (thermal, resonance, or fast), until a practicable method of achieving gain at excitation far below inversion has been demonstrated.

(3) Pumping with bremsstrahlung.

\section{E. Research needed}

Although a workable concept has yet to appear, the time has come to emphasize practical approaches, with more experimental work guided by the theoretical work suggested below.

1. Experimental

a. Isomer separation

(1) Continue the ${ }^{197} \mathrm{Hg}$ procedure of Sec. VI.E.1 to obtain an isomerically enriched sample.

(2) Investigate chemical scavenging as an alternative way to recover selectively excited isomerium.

(3) Perform selective excitation experiments with other nuclides.

(4) Develop on-line atomic-beam techniques for separating isomers.

(5) Try other cases; e.g., ${ }^{133} \mathrm{Ba}$.

(6) Try other methods; e.g., optical piston (Werij et al., 1984; Dyer, 1986).

\section{b. Suppression of resonant absorption}

Demonstrate reduced nonresonant absorption by means of Mössbauer transmission experiments, first using RF to couple Zeeman substates, followed by experiments on the Coussement level-crossing concept.

\section{c. Borrmann-mode Mössbauer experiments}

Study the anomalous transmission of recoilless gamma radiation from sources in single-crystal hosts, with emphasis on higher-order wave modes; measure their coupling factors.

\section{d. Properties of candidate nuclides}

(1) Using the maximum possible instrumental resolution, search for and measure energies, spins, and separations of close pairs of nuclear levels for possible two-step application.

(2) Measure properties of candidate nuclides proposed for subinversion graser application. Continue search for two-step transfer candidates.

\section{e. Confirmation and extension of the Skorobogatov/Alpatov experiment}

(1) Confirm the energy-level assignments for this nuclide.

(2) Repeat and extend the experiment to elucidate the mechanism for the effect.

(3) Measure time dependence of the "two-photon" component.

(4) Measure angular dependence of the "two-photon" component. 
(5) Repeat this experiment with other nuclides.

\section{Theoretical}

Provide guidance to the experimental work suggested above; in particular, the following.

\section{a. Subinversion lasing}

Analyze the kinetics of inversionless lasing, including the time dependence in establishing the resonantabsorption-free condition.

\section{b. Wave modes in crystals}

Analyze the kinetics of stimulated emission into multiwave modes, including the effects of temperature and crystal defects.

\section{c. Nuclear modeling}

Apply advanced models of nuclear structure to guide a search for candidate nuclides.

\section{d. Thermal modeling}

Analyze conceptual graser systems, using realistic parameters - in particular, those using bremsstrahlung pumping.

\section{e. Nuclear superradiance}

Develop a theory applicable to three-level systems.

\section{Concept elaboration}

Proposed graser systems should include, at least, quantitative and practical specification of nuclear and material properties, identification and proposed solutions to problems of production and assembly, and calculated kinetic and thermal behavior. Too many concepts that have been advanced to solve one particular problem have introduced other problems that are equally difficult to solve.

Note added. Recently, Wilhelmy, Fowler, Rundberg and Taylor at Los Alamos National Laboratory have repeated the experiment of Skorobogatov and Dzevitskii (1995) described in Section VI.C.3. They irradiated $22 \mathrm{mg}$ of ${ }^{124} \mathrm{Te}$ at the Brookhaven National Laboratory High Flux Breeder Reactor with a fluence greater than $10^{20} \mathrm{n} \mathrm{cm}^{-2}$ and produced a BeTe crystal containing about twice the Russian activity level. A Debye temperature of $(390 \pm 20) \mathrm{K}$ was determined for the crystal using Mössbauer spectroscopy. Gamma-ray spectra were measured as a function of crystal temperature, source distance to detector, and source angle to detector. No indication of superradiance was observed. A $2 \sigma$ limit of $6.1 \times 10^{-21} \mathrm{~cm}^{2}$ was obtained for the stimulated emission cross section. This is about a factor of 10 below the claimed signal of Skorobogatov and Dzevitskii.

\section{ACKNOWLEDGMENTS}

The authors gratefully acknowledge helpful conversations and communication with R. Coussement, G. R. Hoy, G. Skorobogatov, and M. S. Weiss.

\section{REFERENCES}

Alpatov, V. G., A. G. Beda, G. E. Bizina, B. E. Davydov, and M. M. Korotkov, 1977, in Proceedings of International Conference on Mössbauer Spectroscopy, Bucharest, edited by D. Barb and D. Tarina (Central Institute of Physics, Bucharest), p. 43.

Alpatov, V. G., G. E. Bizina, B. E. Davydov, B. E. Dzevtskii, G. R. Kartashov, M. Korotkov, G. V. Kostina, A. A. Sadovskii, and G. A. Skorobogatov, 1986, Izv. Akad. Nauk SSSR Ser. Fiz. 50, 2013.

Anderson, J. Y., 1988, in Advances in Laser Science-III, AIP Conf. Proc. No. 172, edited by Andrew C. Tam, James L. Gole, and William C. Stwalley (AIP, New York), p. 68.

Andreev, A. V., O. Yu. Tikhomirov, and M. O. Shaiymkulov, 1987, Zh. Tekh. Fiz. 57, 1782 [Sov. Phys. Tech. Phys. 32, 1066].

Arthur, E. D., and D. C. George, 1988, J. Quant. Spectrosc. Radiat. Transf. 40, 651.

Artna-Cohen, A., 1988, J. Quant. Spectrosc. Radiat. Transf. 40, 663.

Baldwin, G. C., 1979, Nucl. Instrum. Methods 159, 309.

Baldwin, G. C., 1982a, in Lasers in Nuclear Physics, Nuclear Science Research Conference Series III, edited by C. E. Bemis and H. K. Carter (Harwood Academic, London), p. 403. Baldwin, G. C., 1982b, Phys. Rep. 7, 1.

Baldwin, G. C., 1984, in Laser Interaction and Related Plasma Phenomena, edited by H. Hora and G. Miley (Plenum, New York), p. 107.

Baldwin, G. C., 1986, in Advances in Laser Science I, AIP Conf. Proc. No. 146, edited by W. Stwalley and M. Lapp (AIP, New York), p. 6.

Baldwin, G. C., 1988, J. Quant. Spectrosc. Radiat. Transf. 40, 685.

Baldwin, G. C., 1989a, in Raman Spectroscopy, Sixty Years, edited by H. Bist, J. R. Durig, and J. F. Sullivan (Elsevier Science, Amsterdam), p. 243.

Baldwin, G. C., 1989b, Progress Report on subcontract, "Kinetics of a Proposed Neutron-Burst Pumped Gamma-Ray Laser," Baldwin Scientific, Inc., Santa Fe, New Mexico.

Baldwin, G. C., H. M. Clark, D. Hakala, and R. R. Reeves, 1978, in Proceedings of the 12th Informal Conference on Photochemistry, edited by M. J. Kurilo, NBS Special Publication No. 526.

Baldwin, G. C., and M. S. Feld, 1986, J. Appl. Phys. 59, 3665. Baldwin, G. C., and L. A. McNeil, 1977, Los Alamos Internal Report No. LA-7004-MS.

Baldwin, G. C., J. P. Neissel, J. Terhune, and L. Tonks, 1963a, Trans. Am. Nucl. Soc. 6, 178.

Baldwin, G. C., J. P. Neissel, J. Terhune, and L. Tonks, 1963b, Proc. IEEE 51, 1247.

Baldwin, G. C., J. P. Neissel, and L. Tonks, 1966, U.S. Patent 3,324,099.

Baldwin, G. C., J. P. Neissel, and L. Tonks, 1962, General Electric Co. Report No. 62GL22.

Baldwin, G. C., and J. C. Solem, 1979a, Nucl. Sci. Eng. 72, 281. Baldwin, G. C., and J. C. Solem, 1979b, Nucl. Sci. Eng. 72, 290. Baldwin, G. C., and J. C. Solem, 1980, J. Appl. Phys. 51, 2372. 
Baldwin, G. C., and J. C. Solem, 1994, Nuovo Cimento D 16, 627.

Baldwin, G. C., and J. C. Solem, 1995a, Int. J. Laser Phys. 5, 231.

Baldwin, G. C., and J. C. Solem, 1995b, Int. J. Laser Phys. 5, 326.

Baldwin, G. C., J. C. Solem, and V. I. Gol'danskii, 1981, Rev. Mod. Phys. 53, 687.

Baldwin, G. C., and B. Suydam 1977, Los Alamos Internal Report No. LA-UR-77-140.

Balko, B., 1988, J. Quant. Spectrosc. Radiat. Transf. 40, 751.

Balko, B., L. Cohen, and D. A. Sparrow, Eds., 1988, GammaRay Lasers (Pergamon, Oxford), reprinted from J. Quant. Spectrosc. Radiat. Transf. 40, 637.

Balko, B., and I. W. Kay, 1992, Institute for Defense Analyses Report No. IDA-P-2533; IDA/HQ-90-36742.

Balko, B., I. W. Kay, and J. W. Neuberger, 1995, Phys. Rev. B 52, 858.

Balko, B., I. W. Kay, J. D. Silk, and D. A. Sparrow, 1995, Laser Phys. (Moscow) 5, 355.

Bauer, R. W., J. A. Becker, E. A. Henry, and K. E. Sale, 1988, J. Quant. Spectrosc. Radiat. Transf. 40, 637.

Batterman, B. W., and H. Cole, 1961, Rev. Mod. Phys. 36, 681. Berger, J. F., D. Gogny, and M. S. Weiss, 1988, J. Quant. Spectrosc. Radiat. Transf. 40, 717.

Berger, J. F., D. Gogny, and M. S. Weiss, 1991, Phys. Rev. A 43, 455 .

Biedenharn, L., G. Baldwin, K. Boyer, and J. Solem, 1986, in Advances in Laser Science I, AIP Conf. Proc. No. 146, edited by W. Stwalley and M. Lapp, (AIP, New York), p. 52.

Biedenharn, L., K. Boyer, and J. Solem, 1985, Bull. Am. Phys. Soc. 30, 1822.

Biedenharn, L., K. Boyer, and J. Solem, 1986, in Advances in Laser Science I, AIP Conf. Proc. No. 146, edited by W. Stwalley and M. Lapp, (AIP, New York), p. 50.

Bizina, G. E., A. G. Beda, N. A. Burgov, and A. V. Davydov, 1963, Zh. Éksp. Teor. Fiz. 45, 1408 [Sov. Phys. JETP 18, 973]. Bonchev, T., and P. Kamenov 1975, Bulg. J. Phys. II 5, 425. Boolchand, P., 1988, J. Quant. Spectrosc. Radiat. Transf. 40, 777.

Boolchand, P., 1995, Laser Phys. (Moscow) 5, 292.

Boolchand, P., and R. Coussement, 1992, Report on Contract No. N00014-88-2035, Cincinnati University, Ohio, Dept. of Electrical and Computer Engineering.

Boolchand, P., R. Coussement, G. Scheveneels, F. Hardeman, and P. Put, 1988, J. Quant. Spectrosc. Radiat. Transf. 40, 773. Borrmann, G., 1941, Phys. Z. 42, 157.

Bounds, J. A., and P. Dyer, 1992, Phys. Rev. C 46, 852.

Bounds, J. A., P. Dyer, and R. C. Haight, 1987, in Advances in Laser Science II, AIP Conf. Proc. No. 160, edited by William C. Stwalley, Marshall Lapp, and Geraldine A. KenneyWallace (AIP, New York), p. 87.

Bounds, J. A., and R. C. Haight, 1988, Proc. SPIE 875, 88. Bowman, C. D., 1976, proposal and private communication. Bowman, C. D., 1987, 6th International Symposium on Capture Gamma-Ray Spectroscopy, Leuven, Belgium (Katholieke Universiteit, Leuven).

Bowman, C. D., 1989, Los Alamos National Laboratory Patent Application No. PAT-APPL-7-329 710.

Boyer, K., and C. Rhodes, 1985, Phys. Rev. Lett. 54, 1490.

Boyer, K., and C. Rhodes, 1994, J. Phys. B 27, L633.

Boyer, K., B. Thompson, A. McPherson, and C. Rhodes, 1994, J. Phys. B 27, 4373.
Campbell, H. N., 1951, J. Appl. Phys. 22, 1139.

Carroll, J. J., M. J. Byrd, D. G. Richmond, T. W. Sinor, K. N. Taylor, W. L. Hodge, Y. Paiss, C. D. Eberhard, J. A. Anderson, C. B. Collins, E. C. Scarbrough, P. P. Antich, F. J. Agee, D. Davis, G. A. Huttlin, K. G. Kerris, M. S. Litz, and D. A. Whittaker, 1991, Phys. Rev. C 43, 1238.

Carroll, J. J., C. B. Collins, K. Heyde, M. Huber, P. von Neumann-Cosel, V. Y. Ponomarev, D. G. Richmond, A. Richter, C. Schelegel, T. W. Sinor, and K. N. Taylor, 1993, Phys. Rev. C 48, 2238.

Chaplin, D. H., and H. Xu, 1992, University of New South Wales, private communication.

Chirikov, B. V., 1963, Zh. Éksp. Teor. Fiz. 44, 2016 [Sov. Phys. JETP 17, 1355].

Cohen, L., 1988, J. Quant. Spectrosc. Radiat. Transf. 40, 735.

Collins, C., F. Lee, D. Shemwell, and B. DePaola, 1982, J. Appl. Phys. 53, 4645.

Collins, C. B., 1986, in Advances in Laser Science I, AIP Conf. Proc. No. 146, edited by W. Stwalley and M. Lapp (AIP, New York), p. 18.

Collins, C. B., 1987, in Advances in Laser Science II, AIP Conf. Proc. No. 160, edited by William C. Stwalley, Marshall Lapp, and Geraldine A. Kenney-Wallace (AIP, New York), p. 45.

Collins, C. B., 1991, Handbook of Laser Science and Technology (CRC, Boca Raton, FL), p. 561.

Collins, C. B., and J. J. Carroll, 1995, Laser Phys. (Moscow) 5, 209.

Collins, C. B., J. J. Carroll, Y. T. Oganessian, and S. A. Karamian, 1995, Laser Phys. (Moscow) 5, 280.

Collins, C. B., J. J. Carroll, K. N. Taylor, T. W. Sinor, C. Hong, J. Standifird, and D. G. Richmond, 1992, in Laser Interaction and Related Plasma Phenomena (Plenum, NY), Vol. 10, p. 151.

Collins, C. B., J. J. Carroll, K. N. Taylor, T. W. Sinor, C. Hong, J. D. Standifird, and D. G. Richmond, 1993, Laser Part. Beams 11, 43.

Coussement, R., P. Boolchand, G. Scheveneels, F. Hardeman, and P. Put, 1988, J. Quant. Spectrosc. Radiat. Transf. 40, 773.

Coussement, R., G. Neyens, M. Van Den Bergh, and P. Boolchand, 1995, Laser Physics (Moscow) 5, 292.

Coussement, R., M. Van den Bergh, G. S'heeren, G. Neyens, and R. Nouwen, 1993, Phys. Rev. Lett. 71, 1824.

Davenloo, F., E. M. Juengerman, D. R. Jander, T. J. Lee, and C. B. Collins, 1990, J. Appl. Phys. 67, 2081.

Deslattes, R., 1968, Appl. Phys. Lett. 12, 135.

Dicke, R. H., 1954, Phys. Rev. 93, 99.

Dwight, H., 1949, Tables of... Mathematical Data, 807.3, 813.3 (MacMillan, New York)

Dyer, P., 1986, in Advances in Laser Science I, AIP Conf. Proc. No. 146, edited by W. Stwalley and M. Lapp (AIP, New York), p. 33.

Dyer, P. and Baldwin, G. C., 1982, in Lasers in Nuclear Physics, edited by C. E. Bemis and H. K. Carter (Harwood Academic, New York/London), p. 431.

Dyer, P., G. C. Baldwin, A. M. Kittrell, E. Abramson, and D. G. Imre, 1985a, Appl. Phys. Lett. 42, 311.

Dyer, P., G. C. Baldwin, A. M. Sabbas, C. Kittrell, E. L. Schweitzer, E. Abramson, and D. G. Imre, 1985b, J. Appl. Phys. 58, 2431.

Eerckens, J. W., 1969, U.S. Patent 3,430,046.

Einstein, A., 1917, Phys. Z. 18, 121.

Eliezer, S., J. M. Martinez-Val, and J. L. Borowitz, 1995, Laser Phys. (Moscow) 5, 323. 
Eliezer, S., J. M. Martinez-Val, Y. Paiss, and G. Velarde, 1995, Kvant. Elektron. (Moscow) 22, 1140.

Elton, R. C., 1990, X-Ray Lasers (Academic, New York), p. 216.

Ewald, P. P., 1913, Phys. Z. 14, 465.

Fearn, H., M. O. Scully, S. Y. Zhu, and M. Sargent III, 1992, Z. Phys. D 22, 495.

Fill, E. E., H. Fearn, C. Keitel, M. O. Scully, and S. Y. Zhu, 1992, Opt. Commun. 87, 323.

Frauenfelder, H., 1960, The Mössbauer Effect (Benjamin, New York).

Friar, J. L., and H. R. Reiss, 1987, Phys. Rev. C 36, 283.

Gadomskii, O. N., and K. V. Krutitskii, 1995, Laser Phys. (Moscow) 5, 379.

Gao, J., C. Guo, X. Guo, G. Jin, P. Wang, J. Zhao, H. Zhang, Y. Jiang, D. Wang, and D. Jiang, 1992, Opt. Commun. 93, 323.

Gol'danskii, V. I., and Y. M. Kagan, 1973, Sov. Phys. JETP 37, 49.

Gol'danskii, V. I., Y. M. Kagan, andV. A. Namiot, 1973, Zh. Éksp. Teor. Fiz. Pis'ma Red. 18, 34 [JETP Lett. 18, 34].

Gol'danskii, V. I., S. V. Karyagin, and V. A. Namiot, 1974, Zh.

Éksp. Teor. Fiz. Pis'ma Red. 19, 625 [JETP Lett. 19, 325].

Gol'danskii, V. I., R. N. Kuz'min, and V. A. Namiot, 1981a, in Mössbauer Spectroscopy II, edited by U. Gonser, Topics in Current Physics No. 5 (Springer, Berlin), p. 49.

Gol'danskii, V., R. N. Kuz'min, and V. Namiot, 1981b, in Proceedings of the International Conference on Lasers '81, edited by C. Collins (STS, McLean, VA), p. 288.

Greaves, E. D., L. Sajo-Bohus, and H. Groening, 1987, Nuovo Cimento D 9, 1303.

Gross, M., and S. Haroche, 1982, Phys. Rep. 93, 301.

Haake, F., and R. Reibold, 1984, Phys. Rev. A 29, 3208.

Hannon, J. P., and G. T. Trammel, 1975, Opt. Commun. 15, 330.

Harris, S. E., 1989, Phys. Rev. Lett. 62, 1033.

Hartmann, F. X., D. W. Noid, and Y. Y. Sharon, 1995, Laser Phys. (Moscow) 5, 371.

Hartmann, F. X., S. R. Rotman, and K. K. Garcia, 1988, J. Quant. Spectrosc. Radiat. Transf. 40, 701.

Heitler, W., The Quantum Theory of Radiation, 2nd Ed. 1944 (Oxford University, New York),

Holcomb, C., and D. A. Sparrow, 1995, Laser Phys. (Moscow) 5, 403.

Hopf, F., P. Meistre, M. Scully, and J. Seely, 1975, Phys. Rev. Lett. 35, 511.

Howerton, R., 1982, The 1982 Version of Evaluated Nuclear Structure Libraries ENSL and CDRL, Lawrence Livermore Laboratory Report No. UCRL-50400, Volume 23, Addendum [based on the compilation given in Table of Isotopes, 7th Ed., edited by C. M. Lederer and V. S. Shirley, 1978 (Wiley, New York)].

Hoy, G. R., 1986, in Advances in Laser Science I, AIP Conf. Proc. No. 146, edited by W. Stwalley and M. Lapp (AIP, New York), p. 54.

Hoy, G. R., and R. D. Taylor, 1988, J. Quant. Spectrosc. Radiat. Transf. 40, 763.

Hubbell, J. H., and M. J. Berger, 1965, NBS Report 8681, U.S. Department of Commerce.

Hutton, J. T., 1986, Ph.D. thesis (Rice University).

Hutton, J. T., G. T. Trammell, and J. P. Hannon, 1987, in Advances in Laser Science II, AIP Conf. Proc. No. 160, edited by William C. Stwalley, Marshall Lapp, and Geraldine A. Kenney-Wallace (AIP, New York), p. 55.

Icsevgi, A., and W. R. Lamb, Jr., 1969, Phys. Rev. 185, 517.

Il'inskii, Yu. A., 1978, Priroda (Moscow) 9, 49 [In Russian].

Il'inskii, Yu. A., and R. V. Khokhlov, 1973, Zh. Éksp. Teor.

Fiz. 65, 1619 [Sov. Phys. JETP 38, 8099].

Ishkhanov, B. S., and I. M. Piskarev, 1980, Yad. Fiz. 32, 593.

Izawa, I., and G. Yamanaka, 1979, Phys. Lett. 88B, 59.

Jaegle, P., 1986, Ed., X-Ray Lasers, J. Phys. (Paris), Colloq. C6, 47.

James, R. W., 1950, Optical Principles of the Diffraction of $X$-Rays (Bell, London).

Josephson, B. D., 1960, Phys. Rev. Lett. 4, 341.

Kagan, Y. M., and A. M. Afanas'ev, 1964, Zh. Éksp. Teor. Fiz. 48, 327 [Sov. Phys. JETP 21, 215].

Kagan, Yu, 1965, Zh. Eksp. Teor. Fiz. 47, 366 [Sov. Phys. JETP 20, 243 (1965)].

Kalman, P., I. Toth, and A. Toth, 1995, Laser Phys. (Moscow) 5, 401.

Kamenov, P. S., 1988, C. R. Acad. Bulg. Sci. 41, 55.

Kamenov, P. S., 1991, Nuovo Cimento D 13, 1369.

Kamenov, P., and T. Bonchev, 1975, C. R. Acad. Bulg. Sci. 28, 1175.

Kamenov, P. S., I. D. Christoskov, A. Petrakiev, and K. P. Kamenov, 1995, Laser Phys. (Moscow) 5, 407.

Kamenov, P. S., and A. Petrakiev, 1992, Bulg. J. Phys. 19, 31.

Kamenov, P. S., A. P. Petrakiev, and A. V. Apostolov, 1995, Laser Phys. (Moscow) 5, 307.

Kamenov, P. S., A. P. Petrakiev, and K. Kamenov, 1995, Laser Phys. (Moscow) 5, 314.

Karyagin, S. V., 1980, Zh. Éksp. Teor. Fiz. 79, 730 [Sov. Phys. JETP 52, 381].

Karyagin, S. V., 1995, Laser Phys. (Moscow) 5, 343.

Khokhlov, R. V., and Y. A. Il'inskii, 1973, in Nonlinear Processes in Optics, edited by R. E. Soloukhin and G. V. Krivoschokov (USSR Acad. Sci., Siberian Branch, Novosibirsk), p. X.

Kirischuk, V. I., 1994, Institute for Nuclear Research, Ukraine, private communication.

Kirischuk, V. I., 1995, Laser Phys. (Moscow) 5, 268.

Kirischuk, V. I., and L. P. Sidorenko, 1995, Laser Phys. (Moscow) 5, 318.

Kocharovskaya, O., and Ya. Khanin, 1988, Pis'ma Zh. Éksp. Teor. Fiz. 48, 581 [JETP Lett. 48, 630].

Kocharovskaya, O. A., 1995, Laser Phys. (Moscow) 5, 284.

Lamb, W. E., 1939, Phys. Rev. 55, 197.

Lambropoulos, P., 1985, Phys. Rev. Lett. 55, 2141.

Lederer, C. M., and V. S. Shirley, 1978, Eds., Table of Isotopes,

7th Ed. (Wiley, New York).

Letokov, V. S., 1973, Science 188, 451.

Lipkin, H. J., 1960, Ann. Phys. (N.Y.) 9, 332.

Lynch, F. J., R. E. Holland, and M. Hamermesh, 1960, Phys.

Rev. 120, 513.

MacGillivray, J. C., and M. S. Feld, 1976, Phys. Rev. A 14, 1174.

Maiman, T., 1960, Nature 187, 493.

Mandel, L., and E. Wolf, 1965, Rev. Mod. Phys. 37, 213.

Madland, D. G., and D. D. Strottman, 1988, Proc. SPIE 875, 103.

Marcuse, D., 1963, Proc. IEEE 51, 849.

Mazur, V. M., Z. M. Bigan, and I. V. Sokolyuk, 1995, Laser Phys. (Moscow) 5, 273.

Melissinos, A. C., and S. P. Davis, 1959, Phys. Rev. 115, 137. 
Morita, M., 1973, Prog. Theor. Phys. 49, 1574.

Mössbauer, R. L., 1958, Z. Phys. 151, 143.

Oganessian, Y. T., and S. A. Karamian, 1995, Laser Phys. (Moscow) 5, 336.

Olariu, S., I. I. Popescu, C. B. Collins, and N. Ceausescu, 1982,

Rev. Roum. Phys. 27, 559.

Otozai, K., R. Arakawa, and M. Morita, 1973, Prog. Theor. Phys. 50, 1771.

Podgoretskii, M. I., and I. I. Rosen, 1960, Zh. Eksp. Teor. Fiz. 39, 1473, [Sov. Phys. JETP 12, 1023 (1961)].

Pound, R., and G. Rebka, 1960, Phys. Rev. Lett. 4, 274.

Reiss, H. R., 1986, in Advances in Laser Science I, AIP Conf. Proc. No. 146, edited by W. Stwalley and M. Lapp (AIP, New York), p. 68.

Rezaie-Serej, S., G. R. Hoy, and R. D. Taylor, 1995, Laser Phys. (Moscow) 5, 240.

Rhodes, C. 1985, Science 30, 1345.

Ring, P., and P. Schuck, 1980, The Nuclear Many-Body Problem (Springer, New York), Ch. 3.

Rivlin, L. A., 1961, USSR patent disclosure.

Rivlin, L. A., 1991, Kvantovaya Elektron (Moscow) 18, 651

[Sov. J. Quantum Electron. 21, 593].

Rivlin, L. A., 1995, Laser Phys. (Moscow) 5, 297.

Rivlin, L. A., 1996, private communication.

Sadykov, E. K., and A. G. Isavnin, 1995, Laser Phys. (Moscow) 5, 411.

Schwenn, H., 1978, M. S. thesis (Rensselaer Polytechnic Institute, Troy, N.Y.).

Scully, M. O., and M. Fleischhauer, 1994, Science 263, 337.

Scully, M. O., and S. Y. Zhu, 1990, Opt. Commun. 77, 36.

Scully, M. O., S.-Y. Zhu, and A. Gavridiles, 1989, Phys. Rev. Lett. 62, 2813.

Sharpe, R. S., and R. A. Schmitt, 1959, Internal Report No. GA910, General Atomics Corp., San Diego.

Shenoy, G. K., and F. E. Wagner, 1978, Mössbauer Isomer Shifts (North-Holland, Amsterdam).

Skorobogatov, G. A., 1975, Phys. Lett. A 53, 72.

Skorobogatov, G. A., 1980, Zh. Tekh. Fiz. 50, 1731.

Skorobogatov, G. A., 1990, Nuovo Cimento D 12, 793.

Skorobogatov, G. A., and B. E. Dzevitskii, 1973, Dokl. Akad.

Nauk SSSR 212, 1332.

Skorobogatov, G. A., and B. E. Dzevitskii, 1995, Laser Phys. (Moscow) 5, 258.

Smirnov, G. V., Yu. V. Shvyd'ko, and E. Realo, 1984, Pis'ma Zh. Éksp. Teor. Fiz. 39, 33 [JETP Lett. 39, 41].

Solem, J. C., 1979, Los Alamos Internal Report No. LA-7898MS.

Solem, J. C., 1985, Bull. Am. Phys. Soc. 30, 1809.

Solem, J. C., 1986a, in Advances in Laser Science I, AIP Conf. Proc. No. 146, edited by W. Stwalley and M. Lapp (AIP, New York), p. 22.

Solem, J. C., 1986b, J. Opt. Soc. Am. B 3, 1551.

Solem, J. C., 1988, J. Quant. Spectrosc. Radiat. Transf. 40, 713. Solem, J. C., and G. C. Baldwin, 1982, Science 218, 229.

Solem, J. C., and G. C. Baldwin, 1995, Nuovo Cimento D 17, 1131.

Solem, J. C., and L. Biedenharn, 1987, "Primer on Coupling Collective Electronic Oscillations to Nuclei," 1987, Los Alamos National Laboratory Report No. LA-10878.

Solem, J. C., and L. Biedenharn, 1988, J. Quant. Spectrosc. Radiat. Transf. 40, 707.

Solem, J. C., and G. Chapline, 1984, Opt. Eng. (Bellingham) 23, 193.
Sparrow, D. A., B. Balko, and S. Dixit, 1988, J. Quant. Spectrosc. Radiat. Transf. 40, 741.

Storm, E., and H. Israel, 1967, Photon Cross Sections, Los Alamos Internal Report No. LA-3753.

Strottman, D., E. Arthur, and D. Madland, 1986, in Proceedings of the IST/IDA Gamma-Ray Laser Workshop, edited by B. Balko, L. Cohen, and F. Hartmann (Inst. for Defense Analyses, Alexandria, VA), p. 371.

Strottman, D. D., 1988, Proc. SPIE 875, 102.

Stwalley, W., and M. Lapp, 1986, Eds., Advances in Laser Science I, AIP Conf. Proc. No. 146 (AIP, New York), Sec. II.

Terhune, J. H., and G. C. Baldwin, 1965, Phys. Rev. Lett. 14, 589.

Thompson, B., A. McPherson, K. Boyer, and C. Rhodes, 1994, J. Phys. B 27, 4391.

Trammell, G. T., and J. P. Hannon, 1975, Opt. Commun. 15, 325.

Trammell, G. T., J. T. Hutton, and J. P. Hannon, 1988, J. Quant. Spectrosc. Radiat. Transf. 40, 693.

Vali, V., and W. Vali, 1963a, Proc. IEEE 51, 182.

Vali, V., and W. Vali, 1963b, Proc. IEEE 51, 1248.

Van Bürck, U., R. L. Mössbauer, E. Gerdau, R. Rüffer, R. Hollatz, E. V. Smirnov, and J. P. Hannon, 1987, Phys. Rev. Lett. 59, 355.

Van Bürck, U., D. P. Siddons, J. B. Hastings, U. Bergmann, and R. Hollatz, 1992, Phys. Rev. B 46, 6207.

Visscher, W. M., 1960, Ann. Phys. (N.Y.) 9, 194.

Vorontsov, V. I., and V. I. Vysotskii, 1974, Kvantovaya Elektron. 8, 69 [Sov. J. Quantum Electron. 8, 69].

Vysotskii, V. I., 1978, Zh. Éksp. Teor. Fiz. 77, 492 [Sov. Phys. JETP 50, 250].

Vysotskii, V. I., 1979, Sov. Phys. JETP 50, 250.

Vysotskii, V. I., 1980, Kvant. Elektron. (Moscow) 19, 3.

Vysotskii, V. I., and R. N. Kuzmin, 1995, Laser Phys. (Moscow), 5, 301.

Vysotskii, V. I., R. N. Kuzmin, and N. V. Maksyuta, 1995, Laser Phys. (Moscow), 5, 396.

Vysotskii, V. I., and V. I. Vorontsov, 1977, Zh. Éksp. Teor. Fiz. 73, 54 [Sov. Phys. JETP 46, 27].

Vysotskii, V. I., and V. I. Vorontsov, 1982, Kvant. Elektron. (Moscow) 23, 3.

Waugh, J. S., L. M. Huber, and U. Haeberlen, 1968, Phys. Rev. Lett. 20, 182.

Weiss, M. S., 1988, Proc. SPIE 875, 109.

Weisskopf, V., and E. Wigner, 1930, Z. Phys.63, 54.

Werij, H. G., J. P. Woerdman, J. J. Beenaker, and I. Kusker, 1984, Phys. Rev. Lett. 52, 2237.

Wildner, W., and U. Gonser, 1979, J. Phys. (France) 40, C2, 48. Williams, M., 1966, The Slowing-Down and Thermalization of Neutrons, (Wiley, New York), p. 339.

Winterberg, F., 1986, in Advances in Laser Science I, AIP Conf. Proc. No. 146, edited by W. Stwalley and M. Lapp (AIP, New York), p. 67.

Wood, L., and G. Chapline, 1974, Nature (London) 252, 447.

$\mathrm{Xu}$, Huang, 1992, Ph.D. Thesis (University of New South Wales).

Yariv, A., 1967, Quantum Electronics (Wiley, New York).

Zadernovsky, A. A., 1995, Laser Phys. (Moscow) 5, 362.

Zaretskii, D. F., and V. V. Lomonosov, 1965, Zh. Eksp. Teor.

Fiz. 48, 368 [Sov. Phys. JETP 21, 243].

Zibrov, A. S., M. D. Lukin, L. W. Hollberg, D. E. Nikonov, M. O. Scully, Y. L. Velichansky, and H. G. Robinson, 1996, Phys. Rev. Lett. 76, 3935. 